\title{
ON THE STRUCTURE OF SETS WITH POSITIVE REACH
}

\author{
JAN RATAJ, LUDĚK ZAJÍČEK
}

\begin{abstract}
We give a complete characterization of compact sets with positive reach (=proximally $C^{1}$ sets) in the plane and of one-dimensional sets with positive reach in $\mathbb{R}^{d}$. Further, we prove that if $\emptyset \neq A \subset \mathbb{R}^{d}$ is a set of positive reach of topological dimension $0<k<d$, then $A$ has its " $k$-dimensional regular part" $\emptyset \neq R \subset A$ which is a $k$-dimensional "uniform" $C^{1,1}$ manifold open in $A$ and $A \backslash R$ can be locally covered by finitely many (k-1)-dimensional DC surfaces. We also show that if $A \subset \mathbb{R}^{d}$ has positive reach, then $\partial A$ can be locally covered by finitely many semiconcave hypersurfaces.
\end{abstract}

\section{INTRODUCTION}

Federer in his fundamental paper [18 unified the approaches of convex and differential geometry, introducing curvature measures for sets with positive reach and proving the kinematic formulas. Sets with positive reach were also studied under distinct names (e.g., "proximally smooth sets" or "prox-regular sets") in general Hilbert spaces, cf. 8 .

Of course, since sets with positive reach form an important class, there exists a number of interesting results on their structure.

First we mention several results on the structure of "special" sets with positive reach. As far as we know, the first interesting result in this direction is essentially contained in Rechetnyak's 1956 paper 29] published before Federer's seminal work. This result which was proved independently, using modern terminology, in [19, reads as follows:

(A) If $f: \mathbb{R}^{d-1} \rightarrow \mathbb{R}$ is Lipschitz, then its (closed) subgraph has positive reach if and only if $f$ is semiconcave.

The following related result was stated (in other words) without a proof in 18, Remark 4.20]:

(B) If $A \subset \mathbb{R}^{d}$ is a Lipschitz manifold of dimension $0<k<d$ with positive reach, then $A$ is a $k$-dimensional $C^{1,1}$ manifold.

The claims of [18, Remark 4.20] easily imply also a more general result.

(C) If $A \subset \mathbb{R}^{d}$ is a topological manifold of dimension $0<k<d$ with positive reach, then $A$ is a k-dimensional $C^{1,1}$ manifold.

A proof of (C) was given by A. Lytchak, see [25, Proposition 1.4] (even in Riemannian manifolds). It is based on a Federer's unproved claim (which is a consequence of (E) below) and on the theory of length spaces (namely $C A T(\kappa)$ spaces). Proofs of (B) for $k=d-1$ are well-known (cf. [31, p. 3] or [12]); we prove also the general case, see Remark 7.3 below.

The authors were supported by the grant GAČR No. P201/15-08218S. 
The following result on special sets with positive reach was proved in [9]:

(D) If $\emptyset \neq A \subset \mathbb{R}^{2}$ is a connected set with positive reach and empty interior, then $A$ is either a singleton or a 1-dimensional manifold (possibly with boundary) of class $C^{1,1}$.

It is written in 9] that the result (D) "gives a complete characterization of connected sets of positive reach with empty interior in the plane", however it is not true for unbounded sets, see Example 8.11 below. In Section 8 we generalize (D) giving a complete characterization of one-dimensional sets $A \subset \mathbb{R}^{d}$ with positive reach.

For general sets with positive reach, there exist several complete characterizations, e.g. Federer's characterization (Proposition 3.3 below) or Lytchak's characterization for compact sets ([25, Theorem 1.3]). However, the structure of sets with positive reach can be rather complicated, and the above characterizations do not give a satisfactory answer to the question "how their structure can be complicated". Our main aim is to give some partial answers to this (unprecise) question.

In $\mathbb{R}^{2}$, we give (see Section 6) an almost satisfactory answer: we provide a simple complete characterization of the local structure of compact sets with positive reach. This is our first main result.

Our second main result on the structure of general sets $A \subset \mathbb{R}^{d}$ with positive reach is an improvement of a further result claimed by Federer in [18, Remark 4.20]. This result works with sets

$$
A^{(k)}:=\{a \in A: \operatorname{dim}(\operatorname{Nor}(A, a)) \geq d-k\},
$$

where $0 \leq k \leq d$ and $\operatorname{Nor}(A, a)$ denotes the normal cone of $A$ at $a$ :

(E) If $A \subset \mathbb{R}^{d}$ is a set of positive reach of topological dimension $0<k \leq d$, then the set $A \backslash A^{(k-1)}$ is open if $k=d$ and it is a $k$-dimensional $C^{1,1}$ manifold open in $A$ for $k<d$.

We will call $R:=A \backslash A^{(k-1)}$ "the main regular part of $A$ ". Federer proved that $A^{(k-1)}$ is countably $(k-1)$-rectifiable; so

$$
A=R \cup A^{(k-1)}
$$

is a (canonical) decomposition of $A$ to a regular (smooth) $k$-dimensional part $R$ and a remaining $(k-1)$-dimensional part $A^{(k-1)}$.

We slightly improve (E) showing that $R$ is even a "uniform $k$-dimensional $C^{1,1}$ manifold" (see Definition 2.5] (e) and Theorem 7.5).

Further we show (Theorem 7.5 ) that

(F) $\quad A^{(k-1)}=A \backslash R$ can be locally covered by finitely many $(k-1)$-dimensional DC surfaces.

In particular, the set $A^{(k-1)}$ is not only $(k-1)$-dimensional, but it has even locally finite $(k-1)$-dimensional Hausdorff measure.

We obtain (F) as a consequence of the fact that some singular sets of convex functions can be covered by finitely many DC surfaces. The proofs of these results which refine the arguments of [33] are contained in Section 4. In fact, these results on singular sets were originally motivated and obtained during our research in progress with D. Pokorný on WDC sets, which provide a natural generalization of sets with positive reach, see [27. By the same method we prove that a $k$-dimensional 
set of positive reach can be locally covered by finitely many $k$-dimensional DC surfaces.

In case $d=k$ we improve (F) showing that the boundary $\partial A=A^{(d-1)}$ of a set of positive reach in $\mathbb{R}^{d}$ can be locally covered by finitely many semiconvex hypersurfaces. We prove that result (Theorem 5.9) directly, without using results on singularities of convex functions.

Using (E), we also observe (Corollary 7.10) that each $A \subset \mathbb{R}^{d}$ with positive reach has a "smooth part" (of non-constant dimension) which is open and dense in $A$.

\section{Preliminaries}

2.1. Basic definitions. The symbols $B(c, r)$ and $\bar{B}(c, r)$ denote open and closed ball of center $c$ and radius $r$, respectively. We also sometimes use notation $B^{X}(c, r)$ for the ball in the space $X$. The closure of a set $A$ is denoted by $\bar{A} \operatorname{or} \operatorname{cl}(A)$ and the set of isolated points of $A$ by isol $A$. The symbol $[x, y]$ denotes the (closed) segment if $x, y \in \mathbb{R}^{d}$. The symbol $\Pi_{M}$ stands for the metric projection, see (4). We consider only real Banach spaces; the norm is always denoted by $|\cdot|$. By span $M$ we denote the linear span of the set $M$ and by $S_{X}$ the unit sphere in $X$. If $X=W \oplus V$, then $\pi_{W}$ is the projection on $W$ along $V$. If $X$ is a Hilbert space and $V$ is not specified, we mean that $V=W^{\perp}$. If $x \in X$ and $x \in X^{*}$, we set $\left\langle x, x^{*}\right\rangle:=x^{*}(x)$. The scalar product of vectors $x, y$ is also denoted by $\langle x, y\rangle$. If $X$ is a Hilbert space, we identify by the standard way $X$ and $X^{*}$. The symbol $\mathcal{H}^{k}$ stands for the $k$-dimensional Hausdorff measure. For sets $A \subset \mathbb{R}^{d}$, we denote by $\operatorname{dim}(A)$ and $\operatorname{dim}_{H}(A)$ the topological and Hausdorff dimensions, respectively. We use the notation $e_{i}$ for the $i$ th canonical basis vector in $\mathbb{R}^{d}, i=1 \ldots, d$.

A mapping is called $K$-Lipschitz if it is Lipschitz with a (not necessarily minimal) constant $K$.

If $f$ is a real function, we use the abbreviated notation $\{f \leq r\}$ for $\{x \in \operatorname{Dom}(f)$ : $f(x) \leq c\}$. The hypograph and epigraph of $f$ are defined as

$$
\begin{aligned}
\text { hypo } f & :=\{(x, t) \in \operatorname{Dom}(f) \times \mathbb{R}: f(x) \geq t\} \\
\text { epi } f & :=\{(x, t) \in \operatorname{Dom}(f) \times \mathbb{R}: f(x) \leq t\} .
\end{aligned}
$$

If $f$ is defined on an open subset of a normed linear space $X$, we use the notation $f_{+}^{\prime}(x, v)$ for the one sided directional derivative of $f$ at $x$ in direction $v$. If $f$ is, in addition, locally Lipschitz, the generalized directional derivative of $f$ at $x \in \operatorname{Dom}(f)$ is defined as

$$
f^{\circ}(x, v):=\limsup _{y \rightarrow x, t \rightarrow 0_{+}} \frac{f(y+t)-f(y)}{t}, \quad v \in X,
$$

and the Clarke's subgradient of $f$ at $x$ is

$$
\partial f(x):=\left\{u^{*} \in X^{*}:\left\langle u^{*}, v\right\rangle \leq f^{\circ}(x, v) \text { for all } v \in X\right\}
$$

(cf. [6, Section 1.2]).

A mapping $F$ between Banach spaces $X, Y$ is called to be $C^{1,1}$, if it is Fréchet differentiable and the derivative $F^{\prime}: \operatorname{Dom}(F) \rightarrow \mathcal{L}(X, Y)$ is Lipschitz.

2.2. Semiconvex functions, DC functions (mappings) and corresponding surfaces. One of several natural equivalent definitions (cf. [11, Definition 1.1.1 and Proposition 1.1.3]) of semiconcavity reads as follows. We formulate it in the generality we need. 
Definition 2.1. A real function $u$ on an open convex subset $C$ of a finite-dimensional Hilbert space $X$ is called semiconcave with a semiconcavity constant $c \geq 0$ if $u$ is continuous on $C$ and the function $g(x)=u(x)-(c / 2)|x|^{2}$ is concave on $C$.

A real function $v$ on $C$ is called semiconvex (with a semiconvexity constant $c \geq 0$ ) if $-v$ is semiconcave (with a semiconcavity constant $c$ ).

If $u, C$ and $X$ are as in the above definition, then (see, e.g., [11, Proposition 2.1.2 and Corollary 3.3.8])

$$
u \text { is } C^{1,1} \text { if and only if } u \text { is both semiconcave and semiconvex. }
$$

We will need the following extension result.

Lemma 2.2. Let $X$ be a finite-dimensional Hilbert space and $\emptyset \neq P \subset X$ be a bounded set, $K \geq 0$ and $c>0$. Let $\psi$ be a Lipschitz function on $P$ such that for each $p \in P$ there exists a functional $h_{p} \in X^{*}$ such that $\left|h_{p}\right| \leq K$ and

$$
\psi(p+\Delta)-\psi(p)-h_{p}(\Delta) \leq c|\Delta|^{2} \quad \text { whenever } \quad \Delta \neq 0 \text { and } p+\Delta \in P .
$$

Then there exists a Lipschitz functions $F$ on $X$ which is semiconcave and $F \uparrow_{P}=\psi$.

This result easily follows from a more general result [15, Proposition 5.12] in which the extended function is semiconvex with a general modulus $\varphi$. It is wellknown (see e.g. [11, p. 30]) that $u$ is semiconcave with a semiconcavity constant $c$ if and only if $u$ is semiconcave with modulus $\varphi(t)=\frac{c}{2} t$. So, to prove Lemma 2.2 it is sufficient to apply [15. Proposition 5.12] to $f:-\psi$ and modulus $\varphi(t):=c t$.

More general than semiconcave functions are DC functions.

Definition 2.3. Let $X$ be a Banach spaces, $C \subset X$ an open convex set and $Y$ a finite-dimensional Banach space.

(i) A real function on $C$ is called a $D C$ function if it is a difference of two continuous convex functions.

(ii) We say that a mapping $F: C \rightarrow Y$ is DC if $y^{*} \circ F$ is $D C$ for every functional $y^{*} \in Y^{*}$.

Remark 2.4. Let $X, C$ and $Y$ be as in Definition 2.3. Then:

(i) The system of all DC functions on $C$ is clearly a vector space.

(ii) $F: C \rightarrow Y$ is DC if and only if $y^{*} \circ F$ is DC for each $y^{*}$ from a basis of $Y^{*}$. It follows clearly from (i).

(iii) If $X$ is a finite-dimensional Hilbert space, then clearly each semiconcave (semiconvex) function on $C$ is DC.

(iv) If $X$ is a finite-dimensional Hilbert space and $F: C \rightarrow Y$ is $C^{1,1}$, then $F$ is DC. It follows from (2), (iii) and (ii).

Definition 2.5. $\quad$ (a) We say that $A \subset \mathbb{R}^{d}$ is a $D C$ surface of dimension $k$ $(0<k<d)$, if there exist a $k$-dimensional subspace $W$ of $\mathbb{R}^{d}$ and a Lipschitz DC mapping $\varphi: W \rightarrow W^{\perp}=: V$ such that $A=\{w+\varphi(w): w \in W\}$.

Then we will also say that $A$ is a DC surface associated with $V$.

(b) For formal reasons, by a DC surface of dimension $d$ in $\mathbb{R}^{d}$ we mean the whole space $\mathbb{R}^{d}$, and by a DC surface of dimension $k=0$ we mean any singleton in $\mathbb{R}^{d}$.

(c) We say that $A \subset \mathbb{R}^{d}$ is a semiconcave hypersurface, if there exist $v \in S_{\mathbb{R}^{d}}$ and a Lipschitz semiconcave function $g$ on $W:=(\operatorname{span}\{v\})^{\perp}$ such that $A=\{w+g(w) v: w \in W\}$. 
(d) We say that $\emptyset \neq A \subset \mathbb{R}^{d}$ is a $D C$ (resp. $C^{1,1}$ ) manifold of dimension $k,(0<k<d)$, if for each $a \in A$ there exist a $k$-dimensional vector space $W \subset \mathbb{R}^{d}$, an open ball $U$ in $W$ and a DC (resp. $C^{1,1}$ ) mapping $\varphi: U \rightarrow W^{\perp}$ such that $P:=\{w+\varphi(w): w \in U\}$ is a relatively open subset of $A$ and $a \in P$.

(e) If $k=d$ (or $k=0$ ), then we mean by a DC (resp. $C^{1,1}$ ) manifold of dimension $k$ in $\mathbb{R}^{d}$ a nonempty open set (or an isolated set).

(f) We say that a $k$-dimensional $(0<k<d) C^{1,1}$ manifold $A \subset \mathbb{R}^{d}$ is a uniform $k$-dimensional $C^{1,1}$ manifold if there exists $K>0$ such that (independently on $a$ ) each $\varphi: U \rightarrow W^{\perp}$ from (d) can be chosen to be $K$-Lipschitz with $K$-Lipschitz derivative $\varphi^{\prime}$.

Remark 2.6. (i) By Remark 2.4 (iii), each semiconcave hypersurface in $\mathbb{R}^{d}$ is a $(d-1)$-dimensional DC surface.

(ii) Using Remark 2.4 (iv), it is easy to see that each $k$-dimensional $C^{1,1}$ manifold in $\mathbb{R}^{d}$ is a $k$-dimensional DC manifold.

(iii) For a uniform $k$-dimensional $C^{1,1}$ manifold $A$, the dependence of the tangent space $\operatorname{Tan}(A, x)$ on $x \in A$ need not be globally Lipschitz, cf. Example 7.13 (1).

\section{BASIC AND AUXILIARY RESUlTS ON SETS OF POSITIVE REACH}

Given a nonempty set $A \subset \mathbb{R}^{d}$, we denote by $\operatorname{Unp} A$ the set of all points $z \in \mathbb{R}^{d}$ for which the metric projection

$$
\Pi_{A}(z):=\{a \in A: \operatorname{dist}(z, A)=|z-a|\}
$$

is a singleton. Abusing slightly the notation, we shall identify $\Pi_{A}(z)$ with its unique element in such a case.

If $A \subset \mathbb{R}^{d}$ and $a \in A$, we define (with $B(a, 0):=\emptyset$ )

$$
\operatorname{reach}(A, a):=\sup \{r \geq 0: B(a, r) \subset \operatorname{Unp} A\},
$$

and

$$
\operatorname{reach} A:=\inf _{a \in A} \operatorname{reach}(A, a) \text {. }
$$

Obviously, if reach $A>0$, then $A$ is closed. Further, it is easy to show that reach $A=\infty$ if and only if $A$ is closed convex (cf. [18, Remark 4.2]).

By $\operatorname{Tan}(A, a)$ we denote the set of all tangent vectors to $A$ at $a$ (i.e., $u \in$ $\operatorname{Tan}(A, a)$ if and only if $u=0$ or there exist $a \neq a_{i} \in A$ and $r_{i}>0$ such that $a_{i} \rightarrow a$ and $\left.r_{i}\left(a_{i}-a\right) \rightarrow u, i \rightarrow \infty\right)$ which is clearly a closed cone. The normal cone of $A$ at $a$ is defined as the dual cone

$$
\operatorname{Nor}(A, a):=\left\{u \in \mathbb{R}^{d}:\langle u, v\rangle \leq 0 \text { for any } v \in \operatorname{Tan}(A, a)\right\} .
$$

In the following proposition we list some known facts on sets with positive reach.

Proposition 3.1. Assume that $A \subset \mathbb{R}^{d}, a \in A$ and $\operatorname{reach}(A, a)>0$.

(i) The function $x \mapsto \operatorname{reach}(A, x)$ is either identically equal to $\infty$, or finite and 1-Lipschitz on A.

(ii) The tangent cone $\operatorname{Tan}(A, a)$ is convex.

(iii) The multifunction $x \mapsto \operatorname{Nor}(A, x) \cap S_{\mathbb{R}^{d}}$ is upper semicontinuous at a. 
(iv) If $v \in \operatorname{Nor}(A, a)$ and $b \in A$ then

$$
\langle b-a, v\rangle \leq \frac{|b-a|^{2}|v|}{2 \operatorname{reach}(A, a)} .
$$

(v) $\operatorname{Nor}(A, a)$ is nontrivial if and only if $a \in \partial A$.

(vi) If reach $(A, a)>r>0$, then

$$
\operatorname{Nor}(A, a)=\left\{\lambda v: \lambda \geq 0,|v|=r, \Pi_{A}(a+v)=a\right\} .
$$

Proof. Property (i) follows easily from the definition. For (ii), see [18, Theorem 4.8. (12)]. Property (iii) can be deduced from [18, Theorem 4.8. (13)] and (iv) follows from [18, Theorem 4.8. (7), (12)]. Property (v) (which can be easily deduced from (vi) and (iii)) follows immeditely from Corollary 3.6, For (vi), see 18, Theorem 4.8. (12)].

Remark 3.2. If $A \subset \mathbb{R}^{d}$ is compact and $0<s<\operatorname{reach} A$, then $\Pi_{A}$ defines a retraction of the open neighbourhood $A_{s}:=\left\{z \in \mathbb{R}^{d}: \operatorname{dist}(z, A)<s\right\}$ onto $A$ (the continuity, even Lipschitzness, of $\Pi_{A}$ follows from [18, Theorem 4.8 (8)]). Hence, $A$ is a Euclidean neighbourhood retract and it follows that the fundamental group and the homology groups of $A$ are finitely generated (see, e.g., Corollary A.8 of 21]). In particular, both $A$ and $\mathbb{R}^{d} \backslash A$ have only finitely many components.

Using the last property and the Lipschitness of $\Pi_{A}$ on $A_{s}$, it is not difficult to prove that $\partial A$ has also finitely many components and any two points lying in the same component of $\partial A$ can be connected in the boundary $\partial A$ by a rectifiable curve.

We note that, however, $\operatorname{int} A$ may have infinitely many components, see Example 7.12 .

Federer's results of [18] easily imply that

$$
\text { if } A \subset \mathbb{R}^{d} \text { is a set of positive reach, then } \operatorname{dim}(A)=\operatorname{dim}_{H}(A) \text {. }
$$

(This fact follows easily from [18, Remark 4.15 (4), (3), (2)] or, more directly, from Theorem 7.5 below.)

Federer ([18, Remark 4.15 (2)]) also proved that

(6) if $A \subset \mathbb{R}^{d}$ has positive reach and $a \in A$, then $\operatorname{dim}(\operatorname{Tan}(A, a)) \leq \operatorname{dim} A$.

We will repeatedly use also the following important Federer's result (18, Theorem 4.18]).

Proposition 3.3. If $A \subset \mathbb{R}^{d}$ is a closed set and $0<t<\infty$, then the following two conditions are equivalent:

(i) $\operatorname{reach}(A) \geq t$.

(ii) $\operatorname{dist}(b-a, \operatorname{Tan}(A, a)) \leq|b-a|^{2} /(2 t)$ whenever $a, b \in A$.

Lemma 3.4. Let $A \subset \mathbb{R}^{d}$ and $\rho>0$ be given.

(i) If $B$ is a closed ball in $\mathbb{R}^{d}$ of radius $\rho, A \cap B \neq \emptyset$ and reach $(A, x)>\rho$ for any $x \in A \cap B$, then $\operatorname{reach}(A \cap B)>\rho$.

(ii) If reach $A>\rho$ and $A \neq \emptyset$ is contained in some closed ball of radius $\rho$ then $A$ is contractible (and, hence, connected).

Proof. Part (i) follows from a more general statement proved in [28, Lemma 4.3]. Assertion (ii) is a direct consequence of [18, Remark 4.15 (1)]. 
Lemma 3.5. Assume that $A \subset \mathbb{R}^{d}, a \in A$ and $\operatorname{reach}(A, a)>0$. Let $C$ be a closed cone contained in int $(\operatorname{Tan}(A, a)) \cup\{0\}$. Then there exists $r>0$ such that $(a+C) \cap B(a, r) \subset A$.

Proof. Assume, for the contrary, that for any $k \in \mathbb{N}$, there exists $y_{k} \in((a+$ $\left.C) \cap B\left(a, \frac{1}{k}\right)\right) \backslash A$. Passing to a subsequence if necessary, we may assume that $\frac{y_{k}-a}{\left|y_{k}-a\right|} \rightarrow u \in C$. Since $u \in \operatorname{Tan}(A, a)$, there exist $z_{k} \in A$ such that $z_{k} \rightarrow a$ and $\frac{z_{k}-a}{\left|z_{k}-a\right|} \rightarrow u$. We can clearly choose $x_{k} \in\left[y_{k}, z_{k}\right] \cap \partial A$ for each $k \in \mathbb{N}$. It is easy to show that $x_{k} \rightarrow a$ and $\frac{x_{k}-a}{\left|x_{k}-a\right|} \rightarrow u$. By Proposition 3.1 (v) we can take a unit normal vector $v_{k} \in S_{\mathbb{R}^{d}} \cap \operatorname{Nor}\left(A, x_{k}\right), k \in \mathbb{N}$, and note that, by Proposition 3.1(iv),

$$
\left\langle v_{k}, \frac{x_{k}-a}{\left|x_{k}-a\right|}\right\rangle \geq-\frac{\left|x_{k}-a\right|}{2 \operatorname{reach}\left(A, x_{k}\right)} \text {. }
$$

Passing to a subsequence, we may achieve that $v_{k} \rightarrow v \in S_{\mathbb{R}^{d}}$ and Proposition 3.1 implies $v \in \operatorname{Nor}(A, a)$. But then, $\operatorname{since}$ reach $\left(A, x_{k}\right) \rightarrow \operatorname{reach}(A, a)$, the above inequality implies that $\langle v, u\rangle \geq 0$. But this is a contradiction, since $u$ lies in the interior of $\operatorname{Tan}(A, a)$ and $v$ is in the dual convex cone to $\operatorname{Tan}(A, a)$.

Corollary 3.6. Assume that $A \subset \mathbb{R}^{d}, a \in A$ and reach $(A, a)>0$. Then $a \in \operatorname{Int} A$ if and only if $\operatorname{Tan}(A, a)=\mathbb{R}^{d}$.

Lemma 3.7. Let $A \subset \mathbb{R}^{d}$, $\operatorname{reach}(A)>0$, and $1 \leq k \leq d$. Let $H^{k}(A)$ be the set of all $a \in A$ such that $\operatorname{Nor}(A, a)$ contains a halfspace of dimension $k$. Then $H^{k}(A)$ is a closed set.

Proof. Suppose that $a_{i} \rightarrow a$, where $a_{i} \in H^{k}(A)$ for each $i$. Since $A$ is closed, we have $a \in A$. We can clearly for each $i$ find an orthonormal system $v_{i}^{1}, \ldots, v_{i}^{k}$ such that all vectors $\pm v_{i}^{1}, \ldots, \pm v_{i}^{k-1}$ and $v_{i}^{k}$ belong to Nor $\left(A, a_{i}\right)$. Using compactness of $S_{\mathbb{R}^{d}}$, we can (and will) suppose that $v_{i}^{1} \rightarrow v^{1}, \ldots, v_{i}^{k} \rightarrow v^{k}$. Proposition 3.1(iii) easily implies that $\pm v^{1}, \ldots, \pm v^{k-1}$ and $v^{k}$ belong to Nor $(A, a)$, and therefore $a \in H^{k}(A)$.

We will essentially use the following immediate consequence of [10, Proposition 5.2. and 5.3].

Proposition 3.8. Let $A \subset \mathbb{R}^{d}$, $\operatorname{reach}(A)>r>0$ and $a \in A$. Then the distance function $d_{A}:=\operatorname{dist}(\cdot, A)$ is semiconvex on $B(a, r / 2)$ with semiconvexity constant $3 / r$ and

$$
\partial d_{A}(x)=\operatorname{Nor}(A, x) \cap \overline{B(0,1)}, \quad x \in A .
$$

We will also need the following simple lemma.

Lemma 3.9. Let $A \subset \mathbb{R}^{d}$ and $\operatorname{reach}(A)>\rho>0$. Let $a \in A$ and $\operatorname{Tan}(A, a)=\{t u$ : $t \geq 0\}$, where $|u|=1$. Set $P:=\{a-t u: 0<t \leq \rho / 4\}$ and $A^{*}:=A \cup P$. Then $\operatorname{reach}\left(A^{*}\right) \geq \rho / 4$.

Proof. We can and will suppose $a=0$. Suppose to the contrary that there exists $z \in \mathbb{R}^{d}$ with $\operatorname{dist}\left(z, A^{*}\right)<\rho / 4$ and two different points $y_{1}, y_{2} \in \Pi_{A^{*}}(z)$. The case $y_{1}, y_{2} \in \bar{P}$ is clearly impossible, since $\bar{P}$ is convex. If $y_{1}, y_{2} \in A$, then $\operatorname{dist}(z, A)=$ $\operatorname{dist}\left(z, A^{*}\right)<\rho / 4$, which contradicts reach $(A)>\rho>0$. So we can suppose that $y_{1} \in P$ and $y_{2} \in A \backslash\{0\}$. Now, if $\langle z, u\rangle \geq 0$, then $|z-0|<\left|z-y_{1}\right|$, a contradiction. So suppose $\langle z, u\rangle<0$. Clearly $\left|y_{2}\right|<\rho / 2$, which implies $\left\langle y_{2}, u\right\rangle>0$. Indeed, if $\left\langle y_{2}, u\right\rangle \leq 0$, then dist $\left(y_{2}, \operatorname{Tan}(A, 0)\right)=\left|y_{2}\right|$ and so Proposition 3.3 gives $\left|y_{2}\right| \leq$ 
$\left|y_{2}\right|^{2} /(2 \rho)$, a contradiction. Consequently there exists $c \in\left[z, y_{2}\right]$ with $\langle c, u\rangle=0$. Clearly $|c|<\rho / 2$ and $c \in \operatorname{Nor}(A, 0)$. So Proposition 3.1(vi) easily gives $\left|c-y_{2}\right|>|c|$. Therefore

$$
|z-0| \leq|z-c|+|c|<|z-c|+\left|c-y_{2}\right|=\left|z-y_{2}\right|,
$$

which contradicts $0 \in A^{*}$.

\section{Singular Points of CONVEX Functions}

There exists a number of articles which study singularities of convex functions. We will deal with convex functions $f$ on an open convex set $C \subset \mathbb{R}^{d}$. Singular points $x$ of $f$ (i.e., the points of non-differentiability of $f$ ) are usually classified by the dimension of $\partial f(x)$; we use the frequent notation

$$
\Sigma^{k}(f):=\{x \in C: \operatorname{dim} \partial f(x) \geq k\} .
$$

Then $\Sigma^{1}(f)$ is the set of all non-differentiability points of $f$. It is well-known for a very long time that $\Sigma^{d}(f)$ is a countable set. A result of [33] says that, for $1 \leq k<d$ and $A \subset C$, the set $A$ is contained in $\Sigma^{k}(f)$ for some convex $f$ on $C$ if and only if $A$ can be covered by countably many DC surfaces of dimension $d-k$ (note that this result is stronger than that of [1] saying that $\Sigma^{k}(f)$ is a $\left(\mathcal{H}^{d-k}, d-k\right)$ set of class $C^{2}$ ).

Following [11, p. 82] we will also consider sets

$$
\Sigma_{r}^{k}(f)=\{x \in C: \partial f(x) \text { contains a } k \text {-dimensional ball of radius } r>0\} \text {. }
$$

Using e.g. [2, Theorem 4.1, (4.2)], we easily obtain that

$$
\Sigma_{r}^{d}(f) \text { is locally finite for each convex function } f \text { on } C \text { and } r>0,
$$

which is essentially an easy old result (cf. [30, p. 14 below]).

In this section (see Proposition 4.4) we will show, refining slightly the method of [33], that $\Sigma_{r}^{k}(f)$ can be covered by finitely many DC surfaces of dimension $d-k$ for each Lipschitz convex function $f$ on $C, 0<k<d$ and $r>0$. It provides a probably new result, except the case $d=2$ and $k=1$, in which it easily follows from [7, Theorem 3.1]. This result will be essentially applied below and can be of some independent interest. (Let us remark that the fact that $\Sigma_{r}^{k}(f)$ has locally finite $\mathcal{H}^{d-k}$ measure follows from [2, Theorem 4.1, (4.2)].)

Although the above mentioned result about sets $\Sigma_{r}^{k}(f)$ does not hold in infinitedimensional spaces, we prove the basic Lemmas 4.1 and 4.2 in general Banach spaces, since it does not increase the difficulty or length of the exposition and it is possible that they can find applications also in this more general context.

Lemma 4.1. Let $E$ be a Banach space, $L>0$, and $\emptyset \neq M \subset E \times \mathbb{R}$. Let the following condition hold.

$\left(C_{L}\right)$ For each point $m=(e, t) \in M$ there exists $e_{m}^{*} \in E^{*}$ such that $\left|e_{m}^{*}\right| \leq L$ and the inequality $t+e_{m}^{*}(\tilde{e}-e) \leq \tilde{t}$ holds for every $\tilde{m}=(\tilde{e}, \tilde{t}) \in M$.

Then there exists a convex L-Lipschitz function $g$ on $E$ such that $M \subset$ graph $g$.

Proof. For each $m \in M$, choose a corresponding $e_{m}^{*}$ and set

$$
g(x):=\sup \left\{t+e_{m}^{*}(x-e): m=(e, t) \in M\right\}, \quad x \in E .
$$

Choose $\left(e_{0}, t_{0}\right) \in M$. Since, for each $x \in E$ and $m=(e, t) \in M$,

$$
t+e_{m}^{*}(x-e)=t+e_{m}^{*}\left(e_{0}-e\right)+e_{m}^{*}\left(x-e_{0}\right) \leq t_{0}+L\left|x-e_{0}\right|,
$$


$g$ is finite. So, by its definition, $g$ is a convex and $L$-Lipschitz function. Using condition $\left(C_{L}\right)$, we clearly obtain $M \subset \operatorname{graph} g$.

Lemma 4.2. Let $X$ be a Banach space, $X=E \oplus K$, where $\operatorname{dim} E \geq 1$ and $1 \leq \operatorname{dim} K<\infty$. Let $\Omega \subset X$ be an open convex set, $f$ a continuous convex function on $\Omega, L>0$ and $\alpha \in K^{*}$. Set

$$
A_{\alpha, L}:=\left\{x \in \Omega: \alpha=p_{x} \Upsilon_{K} \text { for some } p_{x} \in \partial f(x) \text { with }\left|p_{x}\right| \leq L\right\} .
$$

Then there exists a Lipschitz convex function $g$ on $E$ such that

$$
f(x)-\left\langle\pi_{K}(x), \alpha\right\rangle=g\left(\pi_{E}(x)\right) \quad \text { for each } \quad x \in A_{\alpha, L} .
$$

Proof. For each $x \in A_{\alpha, L}$, choose a corresponding $p_{x} \in \partial f(x)$. Further set

$$
M:=\left\{\left(\pi_{E}(x), f(x)-\left\langle\pi_{K}(x), \alpha\right\rangle\right): \quad x \in A_{\alpha, L}\right\} .
$$

For each $m=(e, t) \in M$ choose $x_{m} \in A_{\alpha, L}$ such that $e=\pi_{E}\left(x_{m}\right)$ and $t=$ $f\left(x_{m}\right)-\left\langle\pi_{K}\left(x_{m}\right), \alpha\right\rangle$, and set $e_{m}^{*}:=p_{x_{m}} \curlyvee_{E} \in E^{*}$.

We will show that the condition $\left(C_{L}\right)$ from Lemma 4.1 holds. To this end, choose arbitrary $m=(e, t) \in M, \tilde{m}=(\tilde{e}, \tilde{t}) \in M$ and set $x:=x_{m}, \tilde{x}:=x_{\tilde{m}}$. Since $p_{x} \in \partial f(x)$, we subsequently obtain

$$
\begin{aligned}
f(\tilde{x})-f(x) & \geq\left\langle\tilde{x}-x, p_{x}\right\rangle=\left\langle\pi_{E}(\tilde{x}-x), e_{m}^{*}\right\rangle+\left\langle\pi_{K}(\tilde{x}-x), \alpha\right\rangle, \\
f(\tilde{x})-\left\langle\pi_{K}(\tilde{x}), \alpha\right\rangle & \geq f(x)+\left\langle\pi_{E}(\tilde{x})-\pi_{E}(x), e_{m}^{*}\right\rangle-\left\langle\pi_{K}(x), \alpha\right\rangle, \\
\tilde{t} & \geq t+e_{m}^{*}(\tilde{e}-e) .
\end{aligned}
$$

Thus the condition $\left(C_{L}\right)$ is satisfied and so by Lemma 4.1 there exists a convex $L$-Lipschitz function $g$ on $E$ such that the inclusion $M \subset$ graph $g$ (which is clearly equivalent to (9)) holds.

Lemma 4.3. Let $X$ be a d-dimensional Hilbert space and $K$ its $k$-dimensional subspace, $1 \leq k<d$. Let $\Omega \subset X$ be an open convex set, $L>0, f$ an L-Lipschitz convex function on $\Omega$, and $\varepsilon>0$. For $x \in \Omega$, set $M_{x}:=\left\{p \uparrow_{K}: p \in \partial f(x)\right\} \subset K^{*}$. Further set

$$
A_{\varepsilon}^{K}:=\left\{x \in \Omega: M_{x} \text { contains an open ball in } K^{*} \text { with radius } \varepsilon\right\}
$$

and

$$
Z_{\varepsilon}^{K}:=\left\{x \in \Omega: f_{+}^{\prime}(x, v)+f_{+}^{\prime}(x,-v)>\varepsilon \quad \text { whenever } \quad v \in K \quad \text { and }|v|=1\right\} .
$$

Then both $A_{\varepsilon}^{K}$ and $Z_{\varepsilon}^{K}$ can be covered by a finite number $N$ of $(d-k)$-dimensional $D C$ surfaces associated with $K$, where $N=N(k, L, \varepsilon)$ depends only on $k, L$ and $\varepsilon$.

Proof. Set $E:=K^{\perp}$. In the first step we will prove that, for each open ball $B \subset K^{*}$, the set $A_{B}:=\left\{x \in \Omega: B \subset M_{x}\right\}$ is contained in a $(d-k)$-dimensional DC surface associated with $K$. Choose functionals $\alpha_{0}, \alpha_{1}, \ldots, \alpha_{k}$ in $B$ such that $\alpha_{i}-\alpha_{0}, i=1, \ldots, k$, form a basis of $K^{*}$. Observe that $\left|\alpha_{i}\right| \leq L$. So $A_{B} \subset A_{\alpha_{i}, L}$ for each $0 \leq i \leq k$ (where $A_{\alpha_{i}, L}$ is defined in Lemma 4.2). Using Lemma 4.2, we obtain Lipschitz convex functions $g_{i}, i=0, \ldots, k$, on $E$ such that

$$
f(x)-\left\langle\pi_{K}(x), \alpha_{i}\right\rangle=g_{i}\left(\pi_{E}(x)\right) \text { for each } \quad x \in A_{B} .
$$

Consequently, for each $x \in A_{B}$, we have

$$
\left\langle\pi_{K}(x), \alpha_{i}-\alpha_{0}\right\rangle=g_{0}\left(\pi_{E}(x)\right)-g_{i}\left(\pi_{E}(x)\right), \quad i=1, \ldots, k .
$$

Since $\alpha_{i}-\alpha_{0}, i=1, \ldots, k$, form a basis of $K^{*}$, it is easy to see (using Remark 2.4(ii)) that $A_{B}$ is contained in a DC surface associated with $K$. 
In the second step observe that $M_{x} \subset B^{K^{*}}(0,2 L)$ for each $x \in \Omega$. Choose a finite $(\varepsilon / 2)$-net $Q$ in $B^{K^{*}}(0,2 L)$ with $N=N(k, L, \varepsilon)$ elements. Then clearly each ball of radius $\varepsilon$ in $B^{K^{*}}(0,2 L)$ contains a ball $B^{K^{*}}(q, \varepsilon / 2)$ with $q \in Q$. So $A_{\varepsilon}^{K} \subset \bigcup_{q \in Q} A_{B(q, \varepsilon / 2)}$ and the assertion on $A_{\varepsilon}^{K}$ follows.

Further observe that $M_{x}$ is convex, and $f_{+}^{\prime}(x, v)=\sup \left\{\langle v, \alpha\rangle: \alpha \in M_{x}\right\}$ for each $x \in \Omega$ and $v \in K$. Consequently, for each $x \in Z_{\varepsilon}^{K}$ and each unit $v \in K$, we have

$$
\begin{aligned}
& \sup \left\{\langle v, \alpha\rangle: \alpha \in M_{x}\right\}-\inf \left\{\langle v, \alpha\rangle: \alpha \in M_{x}\right\} \\
& =\sup \left\{\langle v, \alpha\rangle: \alpha \in M_{x}\right\}+\sup \left\{\langle-v, \alpha\rangle: \alpha \in M_{x}\right\}=f_{+}^{\prime}(a, v)+f_{+}^{\prime}(a,-v)>\varepsilon .
\end{aligned}
$$

Thus the minimal width of $M_{x}$ in the space $K^{*}$ (which can be identified with $\mathbb{R}^{k}$ ) is at least $\varepsilon$, and consequently (see, e.g., [17]) $M_{x}$ contains a ball of radius $\varepsilon /(k+1)$. So

$$
Z_{\varepsilon}^{K} \subset A_{\frac{\varepsilon}{k+1}}^{K}
$$

and the assertion on $Z_{\varepsilon}^{K}$ follows.

Proposition 4.4. Let $X$ be a d-dimensional Hilbert space, $1 \leq k<d$ and $\varepsilon>0$. Let $\Omega \subset X$ be an open convex set, $L>0$ and $f$ an L-Lipschitz convex function on $\Omega$. Denote

a) by $\Sigma_{\varepsilon}^{k}$ the set of all $x \in \Omega$ such that $\partial f(x)$ contains an open $k$-dimensional ball (i.e., a ball in a $k$-dimensional affine subspace of $X$ ) of radius $\varepsilon$ and

b) by $Z_{\varepsilon}^{k}$ the set of all $x \in \Omega$ for which there exists a $k$-dimensional space $K \subset X$ such that

$$
f_{+}^{\prime}(x, v)+f_{+}^{\prime}(x,-v)>\varepsilon \quad \text { whenever } \quad v \in K \quad \text { and }|v|=1 .
$$

Then both $\Sigma_{\varepsilon}^{k}$ and $Z_{\varepsilon}^{k}$ can be covered by a finite number $N$ of $(d-k)$-dimensional $D C$ surfaces, where $N=N(d, k, L, \varepsilon)$ depends only on $d, k, L$ and $\varepsilon$.

Proof. First we will prove the assertion on $Z_{\varepsilon}^{k}$. To this end, choose $k$-dimensional subspaces $K_{1}, \ldots, K_{p}$ of $X$ (where $p=p(d, k, L, \varepsilon)$ ) such that for each $k$-dimensional space $K$ there exists $1 \leq i \leq p$ such that the Hausdorff distance of $K \cap S_{X}$ and $K_{i} \cap S_{X}$ is at most $\frac{\varepsilon}{4 L}$. If $x \in Z_{\varepsilon}^{k}$, choose $K$ by the definition of $Z_{\varepsilon}^{k}$ and find $K_{i}$ as above. For each unit $v \in K_{i}$ then there exists a unit vector $\tilde{v} \in K$ such that $|v-\tilde{v}|<\frac{\varepsilon}{4 L}$. Since the mapping $w \mapsto f_{+}^{\prime}(x, w)$ is $L$-Lipschitz (see, e.g., [3, p. 164 , Proposition 7]), we easily obtain that $f_{+}^{\prime}(x, v)+f_{+}^{\prime}(x,-v)>\varepsilon / 2$. Thus our assertion follows from Lemma 4.3, since $Z_{\varepsilon}^{k} \subset \bigcup_{i=1}^{p} Z_{\varepsilon / 2}^{K_{i}}$.

The assertion on $\Sigma_{\varepsilon}^{k}$ then follows, since $\Sigma_{\varepsilon}^{k} \subset Z_{\varepsilon}^{k}$. Indeed, consider an arbitrary $x \in \Sigma_{\varepsilon}^{k}$ and identify in the usual way $X$ and $X^{*}$. Then there exists a $k$-dimensional space $K \subset X$ and $c \in X$ such that $(c+K) \cap B(c, \varepsilon) \subset \partial f(x)$. Consequently, for each unit vector $v \in K$,

$$
\begin{array}{r}
f_{+}^{\prime}(x, v)+f_{+}^{\prime}(x,-v)=\sup \{\langle v, \alpha\rangle: \alpha \in \partial f(x)\}+\sup \{\langle-v, \alpha\rangle: \alpha \in \partial f(x)\} \\
>\left\langle v, c+\frac{\varepsilon}{2} v\right\rangle+\left\langle-v, c-\frac{\varepsilon}{2} v\right\rangle=\varepsilon
\end{array}
$$

As an immediate corollary we obtain the following result. 
Corollary 4.5. Let $\Omega \subset \mathbb{R}^{d}$ be an open convex set, $f$ a Lipschitz convex function on $\Omega$, and $\varepsilon>0$. Let

$$
\begin{aligned}
& A_{1}:=\left\{x \in \Omega: f_{+}^{\prime}(x, v)+f_{+}^{\prime}(x,-v)>\varepsilon \text { for some } v \in S_{\mathbb{R}^{d}}\right\} \text { and } \\
& A_{2}:=\{x \in \Omega: \operatorname{diam}(\partial f(x))>\varepsilon\} .
\end{aligned}
$$

Then both $A_{1}$ and $A_{2}$ can be covered by finitely many DC hypersurfaces.

\section{Singular points of Sets With Positive Reach}

If $A \subset \mathbb{R}^{d}$ is a set with positive reach and $0 \leq k \leq d$, we set, following Federer ([18, p. 447]),

$$
A^{(k)}:=\{a \in A: \operatorname{dim} \operatorname{Nor}(A, a) \geq d-k\} .
$$

(The points of $A^{(k)}$ are, for $k \neq d$, sometimes called $k$-singular boundary points of $A$ and the symbol $\Sigma^{k}(A)$ is then used instead of $A^{(k)}$; see, e.g., 22. However, we will use Federer's notation.)

Federer proved ([18, p. 447]) that $A^{(k)}$ is countably $k$ rectifiable. Using Proposition 3.8 and results of 33, it is easy to obtain a stronger result. (It will be obtained below as a consequence of more subtle Proposition 5.4, see Remark 5.6.)

Proposition 5.1. If $A \subset \mathbb{R}^{d}$ is a set with positive reach and $1 \leq k \leq d-1$, then $A^{(k)}$ can be covered by coutably many $k$-dimensional DC surfaces.

Remark 5.2. We will improve Proposition 5.1 as follows:

(i) $A^{(d-1)}=\partial A$ can be locally covered by finitely many $(d-1)$-dimensional semiconcave surfaces (Theorem 5.9).

(ii) If $1 \leq k=\operatorname{dim} A$, then

(a) $A^{(k-1)}$ can be locally covered by finitely many $(k-1)$-dimensional DC surfaces (Proposition 5.8) and

(b) $A^{(k)}$ can be locally covered by finitely many $k$-dimensional DC surfaces (Theorem 7.5).

To prove (ii), we will classify the points of $A^{(k)}$ by a "strength of singularity" in a similar (but different) way as in 22. (cf. Remark 5.6 below):

Definition 5.3. If $A \subset \mathbb{R}^{d}$ is a set with positive reach, $0 \leq k<d$ and $\varepsilon>0$, then we denote by $A_{\varepsilon}^{(k)}$ the set of all points of $A$, for which $\operatorname{Nor}(A, a) \cap B(0,1)$ contains a $(d-k)$-dimensional ball of radius $\varepsilon$.

Proposition 5.4. Let $A \subset \mathbb{R}^{d}$ be a set with positive reach, $0 \leq k<d$ and $\varepsilon>0$. Then $A_{\varepsilon}^{(k)}$ can be locally covered by finitely many $k$-dimensional $D C$ surfaces.

Moreover, if $k>0$, reach $A>r>0$ and $a \in A$, then the set $A_{\varepsilon}^{(k)} \cap B(a, r / 2)$ can be covered by finite number $N$ of $k$-dimensional DC surfaces, where $N=N(d, k, \varepsilon)$ depends only on $d, k$ and $\varepsilon$.

Proof. Consider an arbitrary $a \in A$ and denote $d_{A}:=\operatorname{dist}(\cdot, A)$. By Proposition 3.8 the function $g(x):=d_{A}(x)+3(2 r)^{-1}|x|^{2}, x \in B(a, r / 2)$, is convex, which clearly implies that also the function

$$
f(x):=d_{A}(x)+3(2 r)^{-1}|x-a|^{2}, \quad x \in B(a, r / 2)
$$

is convex. Moreover, since $d_{A}$ is 1-Lipschitz, it is easy to see that $f$ is Lipschitz with constant $1+3 / 2=5 / 2$. By the basic properties of Clarke subgradient (see 6 , Corollary 1 of Proposition 2.3.3]) we have $\partial f(x)=\partial d_{A}(x)+(3 / r)(x-a)$ for each 
$x \in B(a, r / 2)$. Hence Proposition 3.8 implies that, for each $x \in A_{\varepsilon}^{(k)} \cap B(a, r / 2)$, $\partial f(x)$ contains a $(d-k)$-dimensional ball of radius $\varepsilon$. So, if $k>0$, Proposition 4.4 implies that $A_{\varepsilon}^{(k)} \cap B(a, r / 2)$ can be covered by a finite number $N$ of $k$-dimensional DC surfaces, where $N=N(d, k, \varepsilon)$. If $k=0$, then (8) easily gives that $A_{\varepsilon}^{(k)}$ is locally finite.

Corollary 5.5. Let $A \subset \mathbb{R}^{d}$ be a compact set with positive reach, $0 \leq k<d$ and $\varepsilon>0$. Then $A_{\varepsilon}^{(k)}$ can be covered by finitely many $k$-dimensional DC surfaces.

Remark 5.6. Since clearly $A^{(k)}=\bigcup_{i=1}^{\infty} A_{1 / i}^{(k)}$, Proposition 5.4 implies Proposition 5.1 .

Remark 5.7. Proposition 5.4 is closely related to a result of 22]. Namely, Hug (22, p. 2]) considers (for $0 \leq k<d$ ) sets

$$
\Sigma^{k}(A, \varepsilon):=\left\{x \in \partial A: \mathcal{H}^{d-1-k}\left(\operatorname{Nor}(A, x) \cap S_{\mathbb{R}^{d}}\right) \geq \varepsilon\right\},
$$

which are closely related to our sets $A_{\varepsilon}^{(k)}$. Namely, it is not difficult to show that each set $\Sigma^{k}\left(A, \varepsilon_{1}\right)$ is contained in some $A_{\varepsilon_{2}}^{(k)}$ and vice versa. Consequently, [22, Theorem 3.2] implies (cf. also [22, Corollary 3.6]) that $A_{\varepsilon}^{(k)}$ has locally finite $\mathcal{H}^{k}$ measure and Proposition 5.4 implies that $\Sigma^{k}(A, \varepsilon)$ can be locally covered by finitely many $k$-dimensional DC surfaces.

Suppose that $A \subset \mathbb{R}^{d}$ is a set with positive reach. Federer ([18, p. 447]) proved the following interesting result:

$$
\text { if } \operatorname{dim}(A)=k \geq 1 \text {, then } A=A^{(k)} \neq A^{(k-1)} \text { and, for } a \in A \backslash A^{(k-1)},
$$

$$
\operatorname{Tan}(A, a) \text { is a } k \text {-dimensional vector space. }
$$

Moreover, Federer claimed without a proof ([18, Remark 4.20]) that, if $\operatorname{dim}(A)=$ $k \geq 1$, then

$$
A^{(k-1)} \text { is closed, and }
$$

$$
A \backslash A^{(k-1)} \text { is a } k \text {-dimensional } C^{1,1} \text { manifold. }
$$

We will prove (12) in the following proposition, and statement (13) will be proved in Theorem 7.5 below.

Proposition 5.8. Let $A \subset \mathbb{R}^{d}$ be a set with positive reach and $\operatorname{dim}(A)=k \geq 1$. Then

(i) $A^{(k-1)}$ is closed and

(ii) $A^{(k-1)}$ can be locally covered by finitely many DC surfaces of dimension $k-1$.

Moreover, if $k>1$, reach $A>r>0$ and $a \in A$, then the set $A^{(k-1)} \cap$ $B(a, r / 2)$ can be covered by a finite number $N$ of $(k-1)$-dimensional $D C$ surfaces, where $N=N(d, k)$ depends only on $d$ and $k$.

Proof. Let $a \in A^{(k-1)}$. By definition of $A^{(k-1)}$, Nor $(A, a)$ is a closed convex cone of dimension at least $d-k+1$. Since $\operatorname{dim} \operatorname{Tan}(A, a) \leq k$ by (6), we obtain that Nor $(A, a)$ contains a vector space of dimension $d-k$. Thus Nor $(A, a)$ clearly contains a halfspace of dimension $d-k+1$. Consequently we obtain that $A^{(k-1)}=$ $H^{d-k+1}(A)$, (where $H^{d-k+1}(A)$ is defined in Lemma 3.7). Thus (i) follows from Lemma 3.7 
Further, it is easy to see that $H^{d-k+1}(A) \subset A_{1 / 2}^{(k-1)}$, and so (ii) follows from Proposition 5.4

Theorem 5.9. Let $A \subset \mathbb{R}^{d}$ be a set with $\operatorname{reach}(A)>r>0$ and $a \in \partial A$. Then there exists a finite system $\mathcal{S}$ of semiconcave hypersurfaces which covers $B(a, r / 2) \cap \partial A$.

Moreover, card $\mathcal{S}=N$, where $N=N(d)$ depends only on $d$.

Proof. Choose a finite $(1 / 4)$-net $F$ in $S_{\mathbb{R}^{d}}$ with the cardinality $N=N(d)$. For each $v \in F$ set

$$
M_{v}:=\left\{z \in \partial A:\left|v-n_{z}\right|<1 / 4 \text { for some unit vector } n_{z} \in \operatorname{Nor}(A, z)\right\} .
$$

By Proposition 3.1 (v), $\partial A=\bigcup_{v \in F} M_{v}$, and so it is sufficient to show that for each $v \in F$, the set $S:=B(a, r / 2) \cap M_{v}$ is a subset of a semiconcave hypersurface. To this end, fix an arbitrary $v \in F$, and for each $z \in M_{v}$, choose some $n_{z}$ from the definition of $M_{v}$. Denote $V:=\operatorname{span}\{v\}$ and $W:=V^{\perp}$. Observe that if $x \in S$, then

$$
\left\langle v, n_{x}\right\rangle=\langle v, v\rangle+\left\langle v, n_{x}-v\right\rangle \geq 3 / 4>0 .
$$

Without any loss of generality, we can suppose that $a=0$ and $v=e_{d}$. We will identify $W=\operatorname{span}\left\{e_{1}, \ldots, e_{d-1}\right\}$ with $\mathbb{R}^{d-1}$. Now consider two arbitrary points $x \in S, y \in S$. Using Proposition 3.1(iv) and $|x-y|<r$, we obtain

$$
\left\langle y-x, n_{x}\right\rangle \leq \frac{|y-x|^{2}}{2 r} \leq \frac{|y-x|}{2} .
$$

Writting $y-x=w_{1}+v_{1}$ with $w_{1} \in W$ and $v_{1} \in V$, (15) and $\left|v-n_{x}\right|<1 / 4$ yield

$$
\begin{aligned}
\left|v_{1}\right|=|\langle y-x, v\rangle|=\mid\left\langle y-x, n_{x}\right\rangle & +\left\langle y-x, v-n_{x}\right\rangle \mid \\
& \leq|y-x| / 2+|y-x| / 4 \leq(3 / 4)\left|v_{1}\right|+(3 / 4)\left|w_{1}\right|,
\end{aligned}
$$

which immediately implies $\left|v_{1}\right| \leq 3\left|w_{1}\right|$. Consequently $S$ is the graph of a 3 Lipschitz function $\psi$ defined on a set $P \subset \mathbb{R}^{d-1}$.

Now fix an arbitrary $p \in P$, denote $x:=(p, \psi(p))$ and define $h_{p} \in W^{*}=\left(\mathbb{R}^{d-1}\right)^{*}$ putting $h_{p}(u):=-\left\langle u, n_{x}\right\rangle /\left\langle v, n_{x}\right\rangle$ for $u \in W$.

Using $\left|n_{x}\right|=1$ and (14), we see that $\left|h_{p}\right| \leq 4 / 3$. Further observe that the graph of $h_{p}$ is orthogonal to $n_{x}$ :

$$
\left\langle n_{x}, \Delta+h_{p}(\Delta) v\right\rangle=\left\langle n_{x}, \Delta-\frac{\left\langle\Delta, n_{x}\right\rangle}{\left\langle v, n_{x}\right\rangle} v\right\rangle=0 \text { for each } \Delta \in W .
$$

Now we will verify the condition (3) from Lemma 2.2. To this end, consider $p$ and $x$ as above and an arbitrary $\Delta \in \mathbb{R}^{d-1}$ such that $p+\Delta \in P$. Set $\omega:=$ $\psi(p+\Delta)-\psi(p)-h_{p}(\Delta)$. Since (3) is trivial for $\omega \leq 0$, we suppose $\omega>0$. Denote

$$
y:=(p+\Delta, \psi(p+\Delta)), \quad z:=\left(p+\Delta, \psi(p)+h_{p}(\Delta)\right) .
$$

Then $y-z=\omega v$ and therefore $\omega=|y-z|$. Further $\left\langle n_{x}, z-x\right\rangle=\left\langle n_{x}, \Delta+h_{p}(\Delta) v\right\rangle=$ 0 by (16). Using also (14) we obtain

$$
\left\langle n_{x}, y-x\right\rangle=\left\langle n_{x}, z-x\right\rangle+\left\langle n_{x}, y-z\right\rangle=\left\langle n_{x}, y-z\right\rangle=\left\langle n_{x}, \omega v\right\rangle>0 .
$$

Further

$$
|y-z|=|\langle v, y-z\rangle| \leq\left|\left\langle n_{x}, y-z\right\rangle\right|+\left|\left\langle v-n_{x}, y-z\right\rangle\right| \leq\left|\left\langle n_{x}, y-z\right\rangle\right|+\frac{1}{4}|y-z| .
$$


Hence, using also (17) and (15), we obtain

$$
\omega=|y-z| \leq \frac{4}{3}\left|\left\langle n_{x}, y-z\right\rangle\right|=\frac{4}{3}\left\langle n_{x}, y-x\right\rangle \leq \frac{4}{3} \frac{|y-x|^{2}}{2 r} .
$$

So, since 3-Lipschitzness of $\psi$ gives $|y-x|=|(\Delta, \psi(p+\Delta)-\psi(p))| \leq 4|\Delta|$, we obtain

$$
\psi(p+\Delta)-\psi(p)-h_{p}(\Delta)=\omega \leq \frac{32}{3} \frac{|\Delta|^{2}}{r}=: c|\Delta|^{2} .
$$

So (3) holds and thus Lemma 2.2 gives that $S$ is a subset of a semiconcave hypersurface.

\section{Sets of Positive ReACH In The Plane}

We start with two lemmas which will be needed later.

Lemma 6.1. Let $\delta, \rho>0$ and $0<\eta<1$ be such that

$$
\rho \eta>\delta \text {. }
$$

Let further $A \subset \mathbb{R}^{2}$ and a vertical segment $S \subset \mathbb{R}^{2}$ of length less or equal to $2 \delta$ be given. Assume that for any $x \in A \cap S$, $\operatorname{reach}(A, x) \geq \rho$ and

$$
\left|\left\langle v, e_{2}\right\rangle\right| \geq \eta|v| \text { whenever } v \in \operatorname{Nor}(A, x) .
$$

Then, the intersection $A \cap S$ is connected.

Proof. First, note that the assumption reach $(A, x) \geq \rho, x \in A \cap S$, implies that $A \cap S$ is closed. Assume, for the contrary, that $A \cap S$ is not connected, i.e., there exist two points $x=\left(x_{1}, x_{2}\right), y=\left(x_{1}, y_{2}\right)$ in $S \cap A$ with $x_{2}<y_{2}$ and such that the open segment $(x, y)$ does not intersect $A$. Then, $x \in \partial A$ and we claim that there exists a vector $v \in \operatorname{Nor}(A, x)$ with $\left\langle v, e_{2}\right\rangle>0$. Indeed, if not, (18) would imply that $e_{2}$ lies in the interior of $\operatorname{Tan}(A, x)$, and Lemma 3.5 would imply that $x+\tau e_{2} \in A$ for sufficiently small $\tau>0$, which would contradict our assumption. So, let $v=\left(v_{1}, v_{2}\right) \in \operatorname{Nor}(A, x)$ be a unit vector with $v_{2}>0$. (18) implies that $v_{2} \geq \eta$ and Proposition 3.1 (iv) yields $\langle y-x, v\rangle \leq|y-x|^{2} /(2 \rho)$, hence, $v_{2} \leq 2 \delta /(2 \rho)$. Putting these estimates of $v_{2}$ together, we obtain $\eta \leq \delta / \rho$, which contradicts our assumption and completes the proof.

Lemma 6.2. Let $\varphi: I \rightarrow \mathbb{R}$ be a function defined on an interval $I \subset \mathbb{R}$ and $A \supset \operatorname{graph} \varphi$. Let $\delta, \rho>0$ and $0<\eta<1$ be such that $2 \delta<\rho \eta$, diam $(\operatorname{graph} \varphi) \leq 2 \delta$ and for any $x \in \operatorname{graph} \varphi$ we have $\operatorname{reach}(A, x)>\rho$ and

$$
\exists v \in \operatorname{Nor}(A, x) \cap S_{\mathbb{R}^{2}}:\left\langle v, e_{2}\right\rangle \geq \eta .
$$

Then $\varphi$ is Lipschitz.

Proof. Consider two different numbers $s_{1}, s_{2} \in I$ and denote

$$
x_{1}=\left(s_{1}, \varphi\left(s_{1}\right)\right), x_{2}=\left(s_{2}, \varphi\left(s_{2}\right)\right), u=\left(u_{1}, u_{2}\right):=\frac{x_{2}-x_{1}}{\left|x_{2}-x_{1}\right|} .
$$

To prove the Lipschitz property of $\varphi$, it is clearly sufficient to prove that $u_{2} \leq \lambda$ for some constant $\lambda<1$ (independent of $\left.s_{1}, s_{2}\right)$. If $v=\left(v_{1}, v_{2}\right) \in \operatorname{Nor}\left(A, x_{1}\right)$ is a unit vector from (19) then by Proposition 3.1 (iv) and since $\left|x_{2}-x_{1}\right| \leq 2 \delta$, we get

$$
\langle u, v\rangle \leq \frac{\left|x_{2}-x_{1}\right|}{2 \rho} \leq \frac{\delta}{\rho} .
$$


Observing that clearly $u_{2} v_{2} \leq\langle u, v\rangle+\left|u_{1} v_{1}\right|$, and using $v_{2} \geq \eta$ and (20), we obtain

$$
u_{2} \leq \frac{\delta}{\rho} \frac{1}{\eta}+\frac{\left|u_{1}\right|}{\eta} \leq \frac{1}{2}+\frac{\left|u_{1}\right|}{\eta} .
$$

If $\left|u_{1}\right| \leq \frac{\eta}{4}$ then $u_{2} \leq \frac{3}{4}$, and if not then $\left|u_{2}\right|=\sqrt{1-u_{1}^{2}} \leq \frac{\sqrt{16-\eta^{2}}}{4}<1$. So it is sufficient to put $\lambda:=\max \left\{\frac{3}{4}, \frac{\sqrt{16-\eta^{2}}}{4}\right\}$.

Definition 6.3. Let $M \subset \mathbb{R}^{2}$ and $r>0$. We say that

(1) $M$ is a $\tilde{T}_{r}^{1}$-set if there exists a Lipschitz semiconcave function $\varphi$ on $(-r, r)$ such that $\varphi(0)=0$ and $M=B(0, r) \cap$ hypo $\varphi$.

(2) $M$ is a $\tilde{T}_{r}^{2}$-set if there exist Lipschitz functions $\psi \leq \varphi$ on $(-r, r)$ such that $\varphi$ is semiconcave, $\psi$ is semiconvex, $\varphi(0)=\psi(0)=0, \varphi^{\prime}(0)=\psi^{\prime}(0)=0$ and $M=B(0, r) \cap$ hypo $\varphi \cap$ epi $\psi$.

(3) $M$ is a $\tilde{T}_{r}^{3}$-set if there exist Lipschitz functions $\psi \leq \varphi$ on $[0, r)$ such that $\varphi$ is semiconcave on $(0, r), \psi$ is semiconvex on $(0, r), \varphi(0)=\psi(0)=0$, $\varphi_{+}^{\prime}(0)=\psi_{+}^{\prime}(0)=0$ and $M=B(0, r) \cap$ hypo $\varphi \cap$ epi $\psi$.

(4) $M$ is of type $T^{i}(i=1,2,3)$ at $x \in M$, if there exists an isometry $G: \mathbb{R}^{2} \rightarrow$ $\mathbb{R}^{2}$ such that $G(x)=0$ and $G(M \cap B(x, r))$ is a $\tilde{T}_{r}^{i}$-set for some $r>0$.

Theorem 6.4. Let $A \subset \mathbb{R}^{2}$ and $a \in A$ be given. Then reach $(A, a)>0$ if and only if one of the following statements holds:

(1) $a$ is an interior point of $A$,

(2) $a$ is an isolated point of $A$,

(3) $A$ is of type $T^{i}$ at a for some $i \in\{1,2,3\}$.

Proof. Clearly, reach $(A, a)>0$ if $a$ is an interior or isolated point of $A$. We shall show that the same is true under $(3)$.

Assume that $A$ is of type $T^{i}$ at $a(i=1,2,3)$. We can assume without loss of generality that $a=0$ and that $A \cap B(0, r)$ is a $\tilde{T}_{r}^{i}$-set for some $r>0$. Consider first the case $i=1$ and let $\varphi$ be the Lipschitz semiconcave function from Definition 6.3. By using [19, Proposition 1.7], we can consider $\varphi$ to be defined, Lipschitz and semiconcave on the whole $\mathbb{R}$, and [19, Theorem 2.3] implies that reach (hypo $\varphi)>0$ (cf. (A) in Introduction). Since $A$ coincides with hypo $\varphi$ on a neighbourhood of 0 , we infer that reach $(A, 0)>0$.

Let now $i=2$ and let $\varphi$ and $\psi$ be as in Definition 6.3. Again, we can assume $\varphi, \psi$ to be defined on $\mathbb{R}$, Lipschitz and semiconcave, semiconvex, respectively $(\psi \leq \varphi$ on $(-r, r))$. Set $\rho:=\min \left\{\frac{r}{2}\right.$, reach $($ hypo $\varphi, 0)$, reach $($ epi $\left.\psi, 0)\right\}$ and take a point $x=\left(x_{1}, x_{2}\right) \in B(0, \rho)$. We distinguish three cases: If $x_{2}>\varphi\left(x_{1}\right)$ then $\Pi_{A}(x)=$ $\Pi_{\text {hypo } \varphi}(x)$, if $x_{2}<\psi\left(x_{1}\right)$ then $\Pi_{A}(x)=\Pi_{\text {epi } \psi}(x)$, and if $\psi\left(x_{1}\right) \leq x_{2} \leq \varphi\left(x_{1}\right)$ then $\Pi_{A}(x)=x$. In all these cases, the metric projection to $A$ is single-valued at $x$ and, hence, $\operatorname{reach}(A, 0) \geq \rho$.

Assume now $i=3$, let $\varphi$ and $\psi$ be as in Definition 6.3 and, again, assume that $\varphi, \psi$ are defined on $\mathbb{R}$. Decreasing $r>0$ if necessary, we can assume that $A \cap B(0, r)$ is contained in the cone $\left\{x:\left\langle x, e_{1}\right\rangle \geq \frac{\sqrt{3}}{2}|x|\right\}$. If $x \in B\left(0, \frac{r}{2}\right)$ lies in the dual cone, $\left\{x:\left\langle x, e_{1}\right\rangle \leq-\frac{1}{2}|x|\right\}$, then, clearly, $\Pi_{A}(x)=0$. Consider the functions

$$
\tilde{\varphi}(x):=\min \{x, \varphi(x)\}, \quad \tilde{\psi}(x):=\max \{-x, \psi(x)\} .
$$


The function $\tilde{\varphi}(\tilde{\psi})$ is clearly semiconcave (semiconvex) and coincides with $\varphi(\psi$, respectively) on $(0, r)$ (see, e.g., [11, Proposition 2.1.5]). Set

$$
\rho:=\min \left\{\frac{r}{2}, \operatorname{reach}(\text { hypo } \tilde{\varphi}), \operatorname{reach}(\text { hypo } \tilde{\psi})\right\}
$$

and consider a point $x=\left(x_{1}, x_{2}\right) \in B(0, \rho)$ such that $x_{1}=\left\langle x, e_{1}\right\rangle \geq-\frac{1}{2}|x|$. Again, we distinguish three cases. If $x_{2} \geq \tilde{\varphi}\left(x_{1}\right)$ then $\Pi_{A}(x)=\Pi_{\text {hypo } \tilde{\varphi}}(x)$ is a singleton. If $x_{2} \leq \tilde{\psi}\left(x_{1}\right)$ then $\Pi_{A}(x)=\Pi_{\text {epi } \tilde{\psi}}(x)$ is again a singleton. If $\tilde{\psi}\left(x_{1}\right)<x_{2}<\tilde{\varphi}\left(x_{1}\right)$, then clearly $x_{1}>0$ and therefore $\Pi_{A}(x)=x$. Thus, $\operatorname{reach}(A, 0) \geq \rho$.

We shall show the other implication. Assume that $a \in A$ and

$$
r_{0}:=\min \{\operatorname{reach}(A, a), 1\}>0,
$$

and let $a$ be neither an interior, nor an isolated point of $A$. Then, $\operatorname{Tan}(A, a)$ is a convex cone that neither reduces to $\{0\}$, nor equals the whole $\mathbb{R}^{2}$ (since then, by Corollary 3.6, $a$ would be an interior point of $A$ ). We shall distinguish three cases.

(i) Let $\operatorname{Tan}(A, a)$ be two-dimensional, i.e., there exists a unit vector $v_{0}$ and an $\eta^{\prime} \in(0,1]$ such that

$$
u \in \operatorname{Tan}(A, a) \Longleftrightarrow\left\langle u, v_{0}\right\rangle \leq-\sqrt{1-\eta^{\prime 2}}|u| .
$$

We can assume without loss of generality that $a=0$ and $v_{0}=e_{2}$. We have then $\operatorname{Nor}(A, 0)=\left\{v:\left\langle v, e_{2}\right\rangle \geq \eta^{\prime}|v|\right\}$. Using the definition of the tangent cone, Lemma 3.5 and Proposition 3.1 (iii), (i), subsequently, fixing any $0<\eta<\eta^{\prime}$, we can find a $0<\delta<r_{0} \eta / 4$ such that

$$
\begin{aligned}
& A \cap B(0, \delta) \subset\left\{x:\left\langle x, e_{2}\right\rangle \leq \sqrt{1-\eta^{2}}|x|\right\}, \\
& B(0, \delta) \cap\left\{x:\left\langle x, e_{2}\right\rangle \leq-\sqrt{1-\eta^{2}}|x|\right\} \subset A, \\
& \left\langle v, e_{2}\right\rangle \geq \eta|v| \text { whenever } x \in A \cap \bar{B}(0, \delta) \text { and } v \in \text { Nor }(A, x), \\
& \text { reach }(A, x)>\frac{r_{0}}{2} \text { whenever } x \in A \cap \bar{B}(0, \delta) .
\end{aligned}
$$

We shall use the notation for vertical lines

$$
\ell(s):=\left\{x \in \mathbb{R}^{2}:\left\langle x, e_{1}\right\rangle=s\right\}, \quad s \in \mathbb{R} .
$$

Lemma 6.1 (with $\rho=r_{0} / 2$ and $S=\ell(s) \cap \bar{B}(0, \delta)$ ), (23) and (24) yield that

$$
A \cap \ell(s) \cap B(0, \delta) \text { is connected whenever }|s|<\delta .
$$

If $|s|<\eta \delta$, then $s \sqrt{1-\eta^{2}} / \eta<\sqrt{\delta^{2}-s^{2}}$ and an elementary computation shows that (21) and (22) imply

$$
\{s\} \times\left(-\sqrt{\delta^{2}-s^{2}},-s \eta^{*}\right] \subset A \cap \ell(s) \cap B(0, \delta) \subset\{s\} \times\left(-\sqrt{\delta^{2}-s^{2}}, s \eta^{*}\right],
$$

where $\eta^{*}:=\sqrt{1-\eta^{2}} / \eta$. So, fixing any $0<r<\eta \delta / 4$, we obtain that the function

$$
\varphi(s):=\sup \{t \in \mathbb{R}:(s, t) \in A \cap \ell(s) \cap B(0, \delta)\},|s|<4 r,
$$

is finite and, for each $s \in(-4 r, 4 r)$,

$$
-\sqrt{\delta^{2}-s^{2}}<-s \eta^{*} \leq \varphi(s) \leq s \eta^{*}<\sqrt{\delta^{2}-s^{2}}
$$

which clearly implies that the graph of $\varphi$ is contained in $\partial A \cap B(0, \delta)$. Consequently, due to Proposition 3.1 (v), (23) and (24), we can apply Lemma 6.2 (with $\rho=r_{0} / 2$ and $I=(-4 r, 4 r))$ and get that $\varphi$ is Lipschitz. Set $V_{r}:=\left\{\left(x_{1}, x_{2}\right):\left|x_{1}\right|<4 r\right\}$. Clearly, (25) and (26) imply that

$$
A \cap B(0, \delta) \cap V_{r}=\text { hypo } \varphi \cap B(0, \delta) .
$$


Assume now that $|s|<r$ and $x=(s, \varphi(s))$. We know that $x \in \partial A \cap B(0, \delta)$ and so there exists (see Proposition $3.1(\mathrm{v})$ ) a vector $v \in \operatorname{Nor}(A, x) \cap S_{\mathbb{R}^{2}}$. Proposition 3.1 (vi), (24) and $r<r_{0} / 2$ imply that $B(x+r v, r) \cap A=\emptyset$. It is clear that $B(x+r v, r) \subset V_{r}$. Using $|s|<r<\delta \eta / 4$ and (28), we obtain $|\varphi(s)|<\delta / 4$ and consequently $|x| \leq|s|+|\varphi(s)|<\delta / 2$. Hence, since $r<\delta / 4$, clearly $B(x+r v, r) \subset B(0, \delta)$. Thus $B(x+r v, r) \cap A=\emptyset$ and (29) imply $B(x+r v, r) \cap$ hypo $\varphi=B(x+r v, r) \cap A=\emptyset$ and so, by [19, Theorem 2.6], $\varphi$ is semiconcave on $(-r, r)$. Hence, using also that $\varphi(0)=0$, we get that $A \cap B(0, r)$ is a $\tilde{T}_{r}^{1}$-set and thus $A$ is of type $T^{1}$ at 0 .

(ii) Assume now that $\operatorname{Tan}(A, a)$ is a line; without loss of generality we assume that it is the $x_{1}$-axis and, again, that $a=0$. Hence, Nor $(A, 0)$ is the $x_{2}$-axis and, using the definition of the tangent cone and Proposition 3.1 (iii), we see that for any fixed $\eta \in(0,1)$ there exists a $0<\delta<r_{0} \eta / 4$ such that (24) holds and

$$
\begin{aligned}
& A \cap B(0, \delta) \subset\left\{x:\left|\left\langle x, e_{2}\right\rangle\right| \leq \sqrt{1-\eta^{2}}|x|\right\}, \\
& \left|\left\langle v, e_{2}\right\rangle\right| \geq \eta|v| \text { whenever } x \in A \cap \bar{B}(0, \delta) \text { and } v \in \operatorname{Nor}(A, x) .
\end{aligned}
$$

Lemma 3.4 and (24) yield that $A \cap \bar{B}(0, \delta / 2)$ is connected. This implies that also $\Pi_{1}(A \cap \bar{B}(0, \delta / 2))$ is connected, where $\Pi_{1}$ denotes the orthogonal projection to the $x_{1}$-axis. Since both $e_{1},-e_{1}$ are tangent vectors of $A$ at the origin, $\Pi_{1}(A \cap \bar{B}(0, \delta / 2))$ must contain a neighbourhood of the origin in $\mathbb{R}$ and, so, we can choose an $0<$ $r<\eta \delta / 4$ such that $(-4 r, 4 r) \subset \Pi_{1}(A \cap \bar{B}(0, \delta / 2)) \subset \Pi_{1}(A \cap B(0, \delta))$. Thus, $A \cap \ell(s) \cap B(0, \delta)$ is nonempty if $|s|<4 r$.

Using Lemma 6.1 (again with $\rho=r_{0} / 2$ and $S=\ell(s) \cap \bar{B}(0, \delta)$ ), (24) and (31), we find that the intersection $A \cap \ell(s) \cap B(0, \delta)$ is connected for any $|s|<\delta$. We define the function $\varphi$ on $(-4 r, 4 r)$ again by (27). Thus, using (30), we obtain, similarly as in the case (i), that (28) holds again, and consequently we obtain $\operatorname{graph} \varphi \subset \partial A \cap B(0, \delta)$ again.

We claim that at any point $x \in \operatorname{graph} \varphi$ there exists a unit vector $v \in \operatorname{Nor}(A, x)$ with $\left\langle v, e_{2}\right\rangle \geq \eta$. (Indeed, assume that this is not the case; then, due to (31), all normal vectors $u$ to $A$ at $x$ satisfy $\left\langle u, e_{2}\right\rangle \leq-\eta|u|$ and, hence, $e_{2}$ has to be in the interior of $\operatorname{Tan}(A, x)$. But then, using Lemma [3.5] we get that $\left[x, x+\varepsilon e_{2}\right] \subset A$ for some small $\varepsilon>0$, which contradicts the definition of $\varphi$.) Thus, we may apply Lemma 6.2 (again with $\rho=r_{0} / 2$ and $I=(-4 r, 4 r)$ ) and (24), and get the Lipschitz property of $\varphi$.

We define also

$$
\psi(s):=\inf \{t \in \mathbb{R}:(s, t) \in A \cap \ell(s) \cap B(0, \delta)\},|s|<4 r,
$$

and proceed symmetrically. By the same reasoning, for each $x \in \operatorname{graph} \psi$ there exists a unit vector $v \in \operatorname{Nor}(A, x)$ with $\left\langle v, e_{2}\right\rangle \leq-\eta$, and, applying Lemma 6.2 for the set $A$ reflected by the $x_{1}$-axis, we obtain the Lipschitz property of $-\psi$. Clearly, $\psi \leq \varphi, \varphi(0)=\psi(0)=\varphi^{\prime}(0)=\psi^{\prime}(0)=0$ and

$$
A \cap B(0, \delta) \cap V_{r}=\text { hypo } \varphi \cap \text { epi } \psi \cap B(0, \delta),
$$

where $V_{r}:=\left\{\left(x_{1}, x_{2}\right):\left|x_{1}\right|<4 r\right\}$.

Assume now that $|s|<r$ and $x=(s, \varphi(s))$. We know already that there exists $v \in \operatorname{Nor}(A, x) \cap S_{\mathbb{R}^{2}}$ with $\left\langle v, e_{2}\right\rangle \geq \eta$, hence, since $r<r_{0} / 2, B(x+r v, r) \cap A=$ $\emptyset$ by Proposition 3.1 (vi). By the same argument as in the case (i) we obtain $B(x+r v, r) \subset B(0, \delta) \cap V_{r}$. Since $\left(B(0, \delta) \cap V_{r}\right) \backslash$ hypo $\varphi$ is clearly a component of $\left(B(0, \delta) \cap V_{r}\right) \backslash A$ and the ball $B(x+r v, r) \subset B(0, \delta) \cap V_{r}$ clearly intersects $\left(B(0, \delta) \cap V_{r}\right) \backslash$ hypo $\varphi$ since $\left\langle v, e_{2}\right\rangle \geq \eta$, we get $B(x+r v, r) \cap$ hypo $\varphi=\emptyset$. Thus we 
may apply [19, Theorem 2.6] again and get that $\varphi$ is semiconcave on $(-r, r)$. By a symmetric argument one could verify the semiconvexity of $\psi$ on $(-r, r)$. Hence, $A$ is of type $T^{2}$ at $a$.

(iii) Finally, assume that $\operatorname{Tan}(A, a)$ is a ray. Applying a suitable isometry, we may assume that $a=0$ and $\operatorname{Tan}(A, 0)=\{(s, 0): s \geq 0\}$. Using Lemma 3.9 we get that reach $\left(A \cup\left[-\varepsilon e_{1}, 0\right], 0\right)>0$ if $\varepsilon>0$ is small enough. Clearly, Tan $(A \cup$ $\left.\left[-\varepsilon e_{1}, 0\right], 0\right)$ is the whole $x_{1}$ axis and we may apply the construction from (ii) and get Lipschitz functions $\psi \leq \varphi$ defined on an interval $(-r, r)$ such that $\varphi$ is semiconcave, $\psi$ semiconvex, $\varphi(0)=\psi(0)=\varphi^{\prime}(0)=\psi^{\prime}(0)=0$ and $\left(A \cup\left[-\varepsilon e_{1}, 0\right]\right) \cap B(0, r)=$ (hypo $\varphi \cap$ epi $\psi) \cap B(0, r)$. Then, clearly, $A \cap B(0, r)=\left(\right.$ hypo $\left.\varphi\right|_{[0, r)} \cap$ epi $\left.\left.\psi\right|_{[0, r)}\right) \cap$ $B(0, r)$, thus, $A$ is of type $T^{3}$ at $a$.

Corollary 6.5. A compact set $\emptyset \neq A \subset \mathbb{R}^{2}$ has positive reach if and only if, for each $a \in \partial A \backslash \operatorname{isol} A, A$ is of type $T^{i}$ at a for some $i \in\{1,2,3\}$.

Remark 6.6. If $A \subset \mathbb{R}^{2}$ is a compact set with positive reach, then

$$
\left\{a \in A: A \text { is of type } T^{3} \text { at } a\right\} \text { is finite. }
$$

Indeed, Definition 6.3 and Corollary 6.5 show that each point $x \in A$ has a neighbourhood containing at most one point at which $A$ is of type $T^{3}$.

Remark 6.7. Let $A \subset \mathbb{R}^{d}$ be a connected compact set with positive reach. Lytchak 25, Theorems 1.2, 1.3] proved that every different points $a_{1} \in A, a_{2} \in A$ can be joined in $A$ by a simple $C^{1,1}$ curve. We remind (Remark 3.2) that any two boundary points $b_{1} \in \partial A, b_{2} \in \partial A$ which belong to the same component of $\partial A$ can be joined by a rectifiable curve in $\partial A$ (but clearly not necessarily by a simple $C^{1,1}$ curve). Theorem 6.4 easily implies that in the case $d=2$ such points $b_{1}, b_{2}$ can be joined in $\partial A$ by a more regular curve, e.g. by a curve with finite turn. (For the definition and a theory of curves with finite turn see 14 and the references therein). We do not know whether the statement holds for $d \geq 3$.

\section{Smooth Points of Sets With POSitive ReACH}

To prove that a mapping $\varphi: W \rightarrow V$ is $C^{1,1}$ (with controlled Lipschitz constant of $\varphi^{\prime}$ ), we will use the following special version of "Converse Taylor theorem".

Proposition 7.1. (24], 20]). Let $W, V$ be finite-dimensional Hilbert spaces, $U=B(a, r)$ a ball in $W$ and $\varphi: U \rightarrow V$ a mapping. Suppose that there exists $c>0$ and for each $x \in U$ a linear mapping $g^{x}: W \rightarrow V$ such that

$$
\left|\varphi(y)-\left(\varphi(x)+g^{x}(y-x)\right)\right| \leq c|y-x|^{2} \text { whenever } x, y \in U \text {. }
$$

Then $\varphi \in C^{1,1}(U)$ and $\varphi^{\prime}: U \rightarrow \mathcal{L}(W, V)$ is Lipschitz with constant mc, where $m>0$ is an absolute constant.

Proof. It is sufficient to use [20, Chap. 1, Corollary 126] (with $X:=V, Y:=W$, $k:=1, f:=\varphi$ and $\omega(t):=c t)$, observing that $e_{U}=2$, since $U$ is a ball in $W$.

Proposition 7.2. Let $A \subset \mathbb{R}^{d}$ be a set with reach $A>\rho>0$. Let $W \subset \mathbb{R}^{d}$ be a linear space of dimension $k$, where $1 \leq k \leq d-1$; denote $V:=W^{\perp}$. Let $K>0$, $U \subset W$ be an open ball in $W$ and $\varphi: U \rightarrow V$ be a K-Lipschitz mapping such that $P:=\{w+\varphi(w): w \in U\}$ is a relatively open subset of $A$. Then $\varphi$ is a $C^{1,1}$ mapping and $\varphi^{\prime}: U \rightarrow \mathcal{L}(W, V)$ is Lipschitz with constant $\mu(2+K)^{3} / \rho$, where $\mu$ is an absolute constant. 
Proof. Let $D \subset U$ be the set of all points $w \in U$, for which there exists $\varphi^{\prime}(w)$. It is well-known that $\left|\varphi^{\prime}(w)\right| \leq K$ for each $w \in D$. For $w \in D$, set $g^{w}:=\varphi^{\prime}(w)$. Using the fact that $D$ is dense in $U$ (by Rademacher theorem) and compactness of $\{g \in \mathcal{L}(W, V):|g| \leq K\}$, we can easily to each $x \in U \backslash D$ assign a linear mapping $g^{x} \in \mathcal{L}(W, V)$ with $\left|g^{x}\right| \leq K$ and a sequence $\left(w_{n}^{x}\right) \subset D$ such that $w_{n}^{x} \rightarrow x$ and $\varphi^{\prime}\left(w_{n}^{x}\right)=g^{w_{n}^{x}} \rightarrow g^{x}$. By Proposition 7.1 it is sufficent to verify that (32) holds with $c:=(2+K)^{3} /(2 \rho)$. To this end, consider arbitrary $x, y \in U$.

First consider the case $x \in D$. Set

$a:=x+\varphi(x), \quad L:=\left\{t+g^{x}(t): t \in W\right\}=\left\{t+\varphi^{\prime}(x)(t): t \in W\right\}, \quad M:=a+L$. Clearly, $M=\left\{s+\varphi(x)+\varphi^{\prime}(x)(s-x): s \in W\right\}$. It is a well-known and easy fact that $\operatorname{Tan}(P, a)=L$. Consequently, since $P$ is open in $A, \operatorname{Tan}(A, a)=L$. Set $z_{1}:=y+\varphi(y)$. Using Proposition 3.3 (with $b:=z_{1}, t:=\rho$ ) we obtain

$$
\operatorname{dist}\left(z_{1}, M\right)=\operatorname{dist}\left(z_{1}-a, L\right) \leq \frac{\left|z_{1}-a\right|^{2}}{2 \rho} .
$$

Denote $z_{2}:=y+\varphi(x)+\varphi^{\prime}(x)(y-x) \in M$. Let $p \in M$ with $\left|p-z_{1}\right|=\operatorname{dist}\left(z_{1}, M\right)$ and $w_{p}:=\pi_{W}(p)$. Then

$$
\left|z_{2}-p\right|=\left|\left(y-w_{p}\right)+\left(g^{x}\left(y-w_{p}\right)\right)\right| \leq(1+K)\left|y-w_{p}\right| \leq(1+K)\left|p-z_{1}\right|,
$$

and consequently

$$
\left|z_{2}-z_{1}\right| \leq\left|z_{2}-p\right|+\left|p-z_{1}\right| \leq(2+K)\left|p-z_{1}\right| .
$$

The Lipschitzness of $\varphi$ gives

$$
\left|z_{1}-a\right| \leq|y-x|+|\varphi(y)-\varphi(x)| \leq(1+K)|y-x| .
$$

Using (34), (33) and (35), we obtain

$$
\left|\varphi(y)-\left(\varphi(x)+g^{x}(y-x)\right)\right|=\left|z_{2}-z_{1}\right| \leq(2+K) \cdot \frac{(1+K)^{2}|y-x|^{2}}{2 \rho} \leq c|y-x|^{2},
$$

and so (32) holds if $x \in D$.

In the second case $x \in U \backslash D$, we observe that by (36)

$$
\left|\varphi(y)-\left(\varphi\left(w_{n}^{x}\right)+g^{w_{n}^{x}}\left(y-w_{n}^{x}\right)\right)\right| \leq c\left|y-w_{n}^{x}\right|^{2}, \text { for each } n .
$$

It is easy to see that $g^{w_{n}^{x}}\left(y-w_{n}^{x}\right) \rightarrow g^{x}(y-x)$ as $n \rightarrow \infty$, and so, passing to the limit in (37), we obtain the validity of (32) also in the second case.

Remark 7.3. Proposition 7.2 clearly implies (B) from Introduction.

Proposition 7.4. Let $A \subset \mathbb{R}^{d}$, reach $A>\rho>0$ and $d>k \geq 1$. Suppose that $a \in A, \operatorname{Tan}(A, a)=: W$ is a $k$-dimensional vector space and $\operatorname{dim}(A \cap B(a, \delta))=k$ for some $\delta>0$. Denote $V:=W^{\perp}=\operatorname{Nor}(A, a)$.

Then there exists a ball $U=B^{W}(c, r)$ in $W$ and a $C^{1,1}$ mapping $\varphi: U \rightarrow V$ such that $a=c+\varphi(c), P:=\{w+\varphi(w): w \in U\}$ is a relatively open subset of $A$ and $\varphi^{\prime}$ is $(M / \rho)$-Lipschitz, where $M$ is an absolute constant.

Proof. Set $\omega:=\min \{\rho, \delta / 2\}$ and $A^{*}:=A \cap \bar{B}(a, \omega)$. Then $A^{*}$ has positive reach by Lemma 3.4. We have $\operatorname{dim} A^{*}=k$, since clearly $\operatorname{dim} A^{*} \leq k$ and, by (6), $\operatorname{dim} A^{*} \geq \operatorname{dim} \operatorname{Tan}\left(A^{*}, a\right)=\operatorname{dim} \operatorname{Tan}(A, a)=k$. Since $\left(A^{*}\right)^{(k-1)}$ is closed in $A^{*}$ by Proposition $\left[5.8\right.$ and $a \notin\left(A^{*}\right)^{(k-1)}$, there exists $0<\delta_{0}<\omega$ such that $B\left(a, \delta_{0}\right) \cap A^{*}=B\left(a, \delta_{0}\right) \cap\left(A^{*} \backslash\left(A^{*}\right)^{(k-1)}\right)$. So (11) gives that, if $x \in A \cap B\left(a, \delta_{0}\right)$, then $\operatorname{Tan}(A, x)$ is a $k$-dimensional vector space and so Nor $(A, x)$ is an $(d-k)$-dimensional 
vector space. We can (and will) suppose that $a=0$. Since $V=\operatorname{Nor}(A, 0)$, by Proposition 3.1(iii) we can clearly find $0<\delta_{1}<\delta_{0}$ such that

$$
\operatorname{dist}\left(v, V \cap S_{\mathbb{R}^{d}}\right)<1 / 4 \text { if } v \in \operatorname{Nor}(A, x) \cap S_{\mathbb{R}^{d}} \text { and } x \in B\left(0, \delta_{1}\right) \cap A \text {. }
$$

By [23, Lemma 2], for each $x \in B\left(0, \delta_{1}\right)$ there exists a linear isometry $L: \mathbb{R}^{d} \rightarrow \mathbb{R}^{d}$ such that $L(V)=\operatorname{Nor}(A, x)$ and $L(\operatorname{Nor}(A, x))=V$. Observing also that $L\left(S_{\mathbb{R}^{d}}\right)=$ $S_{\mathbb{R}^{d}}$, it is easy to see that (38) implies

$$
\operatorname{dist}\left(n, \operatorname{Nor}(A, x) \cap S_{\mathbb{R}^{d}}\right)<1 / 4 \text { if } n \in V \cap S_{\mathbb{R}^{d}} \text { and } x \in B\left(0, \delta_{1}\right) \cap A \text {. }
$$

Further choose $0<\delta_{2}<\delta_{1}$ such that

$$
\delta_{2}<\frac{\rho}{8}
$$

Now consider two arbitrary points $x_{1}, x_{2}$ in $A \cap B\left(0, \delta_{2}\right)$ and write $x_{1}=w_{1}+v_{1}$, $x_{2}=w_{2}+v_{2}$, where $w_{1}, w_{2} \in W$ and $v_{1}, v_{2} \in V$. We will show that

$$
\left|v_{1}-v_{2}\right| \leq\left|w_{1}-w_{2}\right| \text {. }
$$

So suppose, to the contrary, that $\left|v_{1}-v_{2}\right|>\left|w_{1}-w_{2}\right|$. Then clearly $\left|v_{1}-v_{2}\right| \geq$ $(1 / 2)\left|x_{2}-x_{1}\right|$. Applying (39) for $n:=\left(v_{2}-v_{1}\right) /\left|v_{2}-v_{1}\right|$, we can choose $n_{1} \in$ Nor $\left(x_{1}, A\right) \cap S_{\mathbb{R}^{d}}$ with $\left|n-n_{1}\right|<1 / 4$. Using Proposition 3.1(iv) and (40), we obtain

$$
\left\langle x_{2}-x_{1}, n_{1}\right\rangle \leq \frac{\left|x_{2}-x_{1}\right|^{2}}{2 \rho} \leq \frac{1}{8}\left|x_{2}-x_{1}\right| .
$$

On the other hand, using $\left|n-n_{1}\right|<1 / 4$ and $\left|v_{1}-v_{2}\right| \geq(1 / 2)\left|x_{2}-x_{1}\right|$, we obtain

$$
\begin{aligned}
& \left\langle x_{2}-x_{1}, n_{1}\right\rangle=\left\langle x_{2}-x_{1}, n\right\rangle+\left\langle x_{2}-x_{1}, n_{1}-n\right\rangle \\
& \quad=\left|v_{2}-v_{1}\right|+\left\langle x_{2}-x_{1}, n_{1}-n\right\rangle \geq \frac{1}{2}\left|x_{2}-x_{1}\right|-\frac{1}{4}\left|x_{2}-x_{1}\right|=\frac{1}{4}\left|x_{2}-x_{1}\right|,
\end{aligned}
$$

which contradicts (42). So (41) holds. Therefore there exists a set $D \subset W$ and a 1-Lipschitz mapping $\psi: D \rightarrow V$ such that

$$
A \cap B\left(0, \delta_{2}\right)=\{w+\psi(w): w \in D\} .
$$

Now we will show that $W \cap B\left(0, \delta_{2} / 8\right) \subset D$, i.e.,

$$
\forall w \in B\left(0, \delta_{2} / 8\right) \cap W \exists v \in V: w+v \in A \cap B\left(0, \delta_{2}\right) .
$$

To this end, fix an arbitrary $w \in B\left(0, \frac{1}{8} \delta_{2}\right) \cap W$, denote $S(w):=w+V$ and suppose, to the contrary, that $S(w) \cap B\left(0, \delta_{2}\right) \cap A=\emptyset$. Then also $S(w) \cap \bar{B}\left(0, \delta_{2} / 2\right) \cap A=\emptyset$ and so we can find $c \in S(w)$ and $d \in \bar{B}\left(0, \delta_{2} / 2\right) \cap A$ such that

$$
|d-c|=\operatorname{dist}\left(S(w), \bar{B}\left(0, \delta_{2} / 2\right) \cap A\right)>0 .
$$

Since $0 \in A$ and $|w|<\delta_{2} / 8$, we have $|c-d|<\delta_{2} / 8$. Obviously $\Pi_{S(w)}(d)=c$, and thus $d-c \in W$. Writting $d=w^{*}+v^{*}$, where $w^{*} \in W$ and $v^{*} \in V$, we obtain

$$
\left|w^{*}\right| \leq|w|+\left|w^{*}-w\right|=|w|+|d-c|<\frac{1}{8} \delta_{2}+\frac{1}{8} \delta_{2}=\frac{1}{4} \delta_{2} .
$$

Since by (41) $\left|v^{*}\right| \leq\left|w^{*}\right|$, we obtain that $|d|<\delta_{2} / 2$, i.e. $d \in B\left(0, \delta_{2} / 2\right)$. Thus, for all sufficiently small $0<t<|d-c|$,

$$
B(d+t(c-d), t|c-d|) \subset B\left(0, \delta_{2} / 2\right),
$$


which clearly implies $\Pi_{A}(d+t(c-d))=d$ and so $n^{*}:=(c-d) /|c-d| \in \operatorname{Nor}(A, d)$ by Proposition 3.1(vi). Since we know that $n^{*} \in W:=V^{\perp}$, we clearly obtain a contradiction with (38).

Applying Proposition 7.2 with $U:=B\left(0, \delta_{2} / 8\right) \cap W, \varphi:=\psi \uparrow_{U}$ and $K=1$, we easily obtain our assertion.

Now we will prove our main theorem on general sets of positive reach in any dimension, which contains Federer's result (13).

Theorem 7.5. Let $A \subset \mathbb{R}^{d}$ be a set of positive reach with $0<k:=\operatorname{dim} A<d$. Then

(i) $A$ can be locally covered by finitely many $D C$ surfaces of dimension $k$.

(ii) $R:=A \backslash A^{(k-1)} \neq \emptyset$ is a uniform $C^{1,1}$ manifold of dimension $k$ which is open in $A$ and $A \backslash R=A^{(k-1)}$ can be locally covered by finitely many DC surfaces of dimension $k-1$.

Proof. To prove (i), observe that for each $a \in A$ (by (6) $) \operatorname{dim} \operatorname{Tan}(A, a) \leq k$ and therefore Nor $(A, a)$ contains a vector space of dimension $d-k$. Consequently clearly $A \subset A_{1}^{(k)}$, and so (i) follows from Proposition 5.4 .

The first part of (ii) follows from Proposition 7.4, since $\operatorname{Tan}(A, a)$ is a $k$-dimensional vector space for each $a \in R:=A \backslash A^{(k-1)}$ by (11). The second part of (ii) was proved in Proposition 5.8 .

Remark 7.6. (To Theorem 7.5)

(a) If $A$ is compact, we can clearly omit (both in (i) and in (ii)) "locally".

(b) Writing "manifolds" instead of "surfaces" we can omit (both in (i) and in (ii)) "locally" also in the case of a non-compact $A$. This follows rather easily from the facts that $N=N(d, k)$ in Proposition 5.8 and $N=N(d, k, \varepsilon)$ in Proposition 5.4 (which is applied, in the proof of (i), with $\varepsilon=1$ ).

(c) It can be shown that $R$ cannot be always locally covered by finitely many $C^{1,1}$ surfaces of dimension $k$ (see Example 7.13).

(d) If $j<k-1$, the set $A^{(j)}$ cannot be always covered by a locally finite system of DC surfaces of dimension $j$. As an example, consider a convex body $A \subset \mathbb{R}^{2}$ with $A^{(0)} \subset \partial A$ infinite.

Further we will consider also relatively open subsets $\emptyset \neq B \subset A$, for which $\operatorname{dim} B \neq \operatorname{dim} A$. We will need the following notation.

Definition 7.7. Let $A \subset \mathbb{R}^{d}$ be a set of positive reach and let $0 \leq k \leq d$. We denote

(a) by $D_{k}(A)$ the set of points $a \in A$, such that $\operatorname{dim}(A \cap B(a, r))=k$ for all sufficiently small $r>0$, and

(b) by $S_{k}(A)$ the set of points $a \in A$, such that $A \cap B(a, r)$ is a $k$-dimensional $C^{1,1}$ manifold for some $r>0$.

Remark 7.8. (i) It is easy to see that $A=\bigcup_{k=0}^{d} D_{k}(A)=\operatorname{isol} A \cup \bigcup_{k=1}^{d} D_{k}(A)$.

(ii) Clearly each $S_{k}(A)$ is open in $A$.

(iii) If $\operatorname{dim} A=k \geq 1$, then

$$
R:=A^{(k)} \backslash A^{(k-1)}=S_{k}(A) .
$$


Indeed, $R \subset S_{k}(A)$ follows from Theorem 7.5 and $S_{k}(A) \subset A^{(k)} \backslash A^{(k-1)}$ follows from the obvious fact that $\operatorname{Tan}(A, x)$ is a $k$-dimensional vector space for each $x \in S_{k}(A)$.

Proposition 7.9. Let $A \subset \mathbb{R}^{d}$ be a set of positive reach and let $1 \leq k \leq d$. Then $S_{k}(A)$ is a dense subset of $D_{k}(A)$.

Proof. Suppose $D_{k}(A) \neq \emptyset$ and choose arbitrary $d \in D_{k}(A)$ and $\delta>0$. Since $d \in$ $D_{k}(A)$, we can choose $0<\omega<\delta$ such that $\omega<\operatorname{reach}(A)$ and $\operatorname{dim}(A \cap B(a, r))=k$ for all $0<r \leq \omega$. By Lemma 3.4, $A^{*}:=A \cap \bar{B}(a, \omega)$ has positive reach. As (e.g., by Proposition 5.1) $\operatorname{dim}\left(\left(A^{*}\right)^{k-1} \cap B(a, \omega / 2)\right) \leq k-1$ and $\operatorname{dim}\left(A^{*} \cap B(a, \omega / 2)\right)=k$, we have $\left(A^{*} \backslash\left(A^{*}\right)^{k-1}\right) \cap B(a, \omega / 2) \neq \emptyset$. Since $A^{*} \backslash\left(A^{*}\right)^{k-1}$ is by Theorem 7.5 a $k$-dimensional $C^{1,1}$ manifold open in $A^{*}$, we easily obtain $S_{k}(A) \cap B(a, \omega / 2) \neq \emptyset$ and (i) follows.

Corollary 7.10. The set of all smooth points $S:=S_{0} \cup S_{1} \cup \cdots \cup S_{d}$ is open and dense in $A$.

However, the structure of the set $A \backslash S$, which is nowhere dense in $A$, can be very complicated and a satisfactory complete characterization even of the local structure of sets of positive reach in $\mathbb{R}^{d}$ for $d \geq 3$ seems to be a very difficult task.

In such spaces we are not able even answer the following natural question:

Question. Let $A \subset \mathbb{R}^{d}$ be a compact set with positive reach. Does there exists a decomposition

$$
A=Q_{1} \cup \cdots \cup Q_{s},
$$

where $Q_{i}, i=1, \ldots, s$, are pairwise disjoint and each $Q_{i}$ is a relatively open subset of a DC surface $P_{i}$ of dimension $0 \leq k_{i} \leq d$ ?

Remark 7.11. (i) Theorem 6.4 implies that for $d=2$ the answer to Question is positive. Indeed, first observe that if each of closed sets $A_{1}, \ldots, A_{k}$ has a decomposition of type (45), then also their union $A_{1} \cup \cdots \cup A_{k}=A_{1} \cup$ $\left(A_{2} \backslash A_{1}\right) \cup \ldots$ has clearly such a decomposition.

Further, observe that Theorem 6.4 easily implies that for each $x \in \partial A$ there exists an open neighbourhood $U_{x}$ of $x$ (e.g. a suitable open square) such that $\partial A \cup \overline{U_{x}}$ has a decomposition of type (45). Using compactness of $\partial A$ and the first observation, we easily conclude the proof.

(ii) If $d \geq 3$, we do not even know whether there always exists a weaker decomposition, in which $Q_{i}$ is supposed to be a relatively open subset of a DC manifold.

(iii) If such a decomposition exists, it is not (clearly) uniquely determined. Moreover, already in the plane in some cases there is no "canonical decomposition" (see Example 7.12).

Example 7.12. Let $\emptyset \neq K \subset \mathbb{R}$ be compact and denote $I:=\operatorname{conv} K$,

$$
\varphi: x \mapsto(\operatorname{dist}(x, K))^{2}, \quad x \in \mathbb{R},
$$

and

$$
A_{K}:=\operatorname{hypo} \varphi \cap \operatorname{epi}(-\varphi) \cap(I \times \mathbb{R}) .
$$

The function $\varphi$ is semiconcave (with semiconcavity constant 2), see 11, Proposition 2.2.2], and, hence, reach $A_{K}>0$ by Corollary 6.5 . 
In what follows, we will identify $\mathbb{R}$ with the $x$-axis $\mathbb{R} \times\{0\}$. The following properties can be easily shown.

(1) $A_{K}$ is topologically regular $\left(A_{K}=\operatorname{cl}\left(\operatorname{int} A_{K}\right)\right)$ if $K$ is totally disconnected, nevertheless, the boundary $\partial A_{K}$ fails to be a 1-dimensional manifold at all points of $K$ (note that $K$ can even have positive one-dimensional measure).

(2) Both $A_{K}$ and $\partial A_{K}$ are (arcwise) connected. However, if $K$ is infinite and totally disconnected, the interior of $A_{K}$ has infinitely many components and the boundary $\partial A_{K}$ is not locally contractible at accumulation points of $K$.

(3) $\partial A_{K} \backslash \partial I$ can be written as the union of two connected one-dimensional DC manifolds which can be chosen in an infinite number of ways. Moreover, there is no "canonical" way how to decompose $\partial A_{K} \backslash \partial I$ into two disjoint one-dimensional DC manifolds (we could choose, e.g., $M_{1}=\left(\partial A_{K} \backslash \partial I\right) \cap$ $\{y \geq 0\}$ and $\left.M_{2}=\partial A_{K} \cap\{y<0\}\right)$.

Example 7.13. Consider the last example with $K$ infinite and totally disconnected to be embedded into the $x, y$ plane in $\mathbb{R}^{3}$. Note that we can write

$$
A_{K}=\mathrm{cl}\left(\bigcup_{i=1}^{\infty} A_{\left\{a_{i}, b_{i}\right\}}\right)
$$

if $\left(a_{i}, b_{i}\right)$ are all the maximal open intervals in $I \backslash K, i=1,2, \ldots$, and that the intersection $A_{\left\{a_{i}, b_{i}\right\}} \cap A_{\left\{a_{j}, b_{j}\right\}}$ is equal to the intersection of the segments $\left[a_{i}, b_{i}\right] \cap$ $\left[a_{j}, b_{j}\right]$ if $i \neq j$. Let $T_{\theta}$ denote the rotation in $\mathbb{R}^{3}$ around the $x$-axis by an angle $\theta \in[0,2 \pi)$ in the positive sense. It is not difficult to show that, choosing any sequence $\Theta=\left(\theta_{i}\right)_{i=1}^{\infty}$, the set

$$
A_{K}^{\Theta}:=\operatorname{cl}\left(\bigcup_{i=1}^{\infty} T_{\theta_{i}}\left(A_{\left\{a_{i}, b_{i}\right\}}\right)\right)
$$

has positive reach and $\operatorname{dim} A_{K}^{\Theta}=2$. Note that the "regular part" of $A_{K}^{\Theta}$ (cf. Theorem 7.5) is

$$
R:=A_{K}^{\Theta} \backslash\left(A_{K}^{\Theta}\right)^{(1)}=\left(A_{K}^{\Theta}\right)^{(2)} \backslash\left(A_{K}^{\Theta}\right)^{(1)}=\bigcup_{i=1}^{\infty} T_{\theta_{i}}\left(\operatorname{int} A_{\left\{a_{i}, b_{i}\right\}}\right) .
$$

We observe the following.

(1) $R$ is a uniformly $C^{1,1} 2$-dimensional manifold (cf. Theorem 7.5 ). Nevertheless, choosing an appropriate sequence $\Theta$, the function

$$
x \mapsto \operatorname{Tan}(R, x)
$$

is not globally Lipschitz on $R$.

(2) Let $x$ be an accumulation point of $K$ and choose a sequence of angles $\left(\theta_{i}\right)$ so that, for any $\delta>0$, the set of those $\theta_{i}$ which correspond to intervals $\left(a_{i}, b_{i}\right) \subset(x-\delta, x+\delta)$ is dense in $[0,2 \pi)$. Then it is easy to see that the regular part $R$ cannot be covered by finitely many $C^{1,1}$ hypersurfaces. (However, $R$, and even $A_{K}^{\Theta}$, can be covered by finitely many DC hypersurfaces by Remark 7.6 (a).) 


\section{One-Dimensional PR SEts}

Definition 8.1 (Intrinsic distance). For $A \subset \mathbb{R}^{d}$ and $x, y \in A$ we set

$$
d_{A}(x, y):=\inf \{\operatorname{length}(\gamma) ; \gamma:[a, b] \rightarrow A \text { continuous, } \gamma(a)=x, \gamma(b)=y\}
$$

and call it intrinsic distance of $x, y$ in $A$.

Remark 8.2. Note that the intrinsic distance may take infinite values, hence, it is not a distance in the standard metric spaces setting. Nevertheless, it clearly has all other properties of a metric (cf. [5, Chapter 1]).

Definition 8.3. Let $k \in\{1, \ldots, d\}$ be given. A set $\emptyset \neq M \subset \mathbb{R}^{d}$ is a $k$-dimensional $C^{1}$ submanifold with boundary if for any $x \in M$ there exist a neighbourhood $U$ of $x$ and a $C^{1}$ diffeomorphism $\phi: M \cap U \rightarrow V$ of $M \cap U$ onto a relatively open subset $V$ of a closed halfspace in $\mathbb{R}^{k}$ (i.e., $\phi$ is a homeomorphism and both $\phi$ and $\phi^{-1}$ are restrictions of $C^{1}$ mappings defined on some open sets). The mapping $\phi$ is called a local $C^{1}$ chart of $M$.

Definition 8.4. A $C^{1,1}$ curve (with parameter $L$ ) is the image of some $C^{1}$ arclength parametrization $\gamma: I \rightarrow \mathbb{R}^{d}$ defined on a (nondegenerate) interval and such that the derivative $\gamma^{\prime}$ is $L$-Lipschitz. We call then $\gamma$ a $C^{1,1}$ arc-length parametrization (with parameter $L$ ). A $C^{1,1}$ curve is

(1) simple if it has a $C^{1,1}$ arc-length parametrization which is a homeomorphism,

(2) closed simple if it has a $C^{1,1}$ arc-length parametrization $\gamma:[a, b] \rightarrow \mathbb{R}^{d}$ such that $\gamma(a)=\gamma(b), \gamma^{\prime}(a)=\gamma^{\prime}(b)$ and $\left.\gamma\right|_{[a, b)}$ is injective,

(3) a simple $C^{1,1}$ arc if it is a compact and simple $C^{1,1}$ curve.

A $C^{1}$ curve (simple $C^{1}$ curve, closed simple $C^{1}$ curve, simple $C^{1}$ arc) is defined as above, but without the Lipschitz property of the derivative.

Remark 8.5. Let $I \subset \mathbb{R}$ be an interval, $\psi: I \rightarrow \mathbb{R}^{d}$ a $C^{1}$ homeomorphism, $\lambda:=$ $\inf \left\{\left|\psi^{\prime}(x)\right|: x \in I\right\}>0$, and let $\psi^{\prime}$ be $M$-Lipschitz. Then $\psi(I)$ is a simple $C^{1,1}$ curve with parameter $2 M / \lambda^{2}$.

Indeed, a standard computation (see., e.g., [16, Lemma 2.7]) reveals that any arc-length reparametrization of $\psi$ has $\left(2 M / \lambda^{2}\right)$-Lipschitz derivative.

Remark 8.6. Let $\gamma: I \rightarrow \mathbb{R}^{d}$ be an arbitrary (not necessary injective) arc-length parametrization $C^{1,1}$ with parameter $L>0$ and $x=\gamma(s), y=\gamma(t)(s, t \in I)$. The mean value theorem for vector valued functions implies (see [13, (8.6.2)])

$$
\left|y-x-\gamma^{\prime}(s)(t-s)\right| \leq L|t-s|^{2} \text {. }
$$

It follows that if $|t-s|<1 /(2 L)$ then (note that $\left|\gamma^{\prime}(s)\right|=1$ )

$$
|y-x| \geq\left|\gamma^{\prime}(s)\right||t-s|-L|t-s|^{2} \geq \frac{1}{2}|t-s|
$$

hence,

$$
\left|y-x-\gamma^{\prime}(s)(t-s)\right| \leq 4 L|y-x|^{2} \text {. }
$$

Lemma 8.7. A connected one-dimensional $C^{1}$ submanifold of $\mathbb{R}^{d}$ with boundary is a simple or closed simple $C^{1}$ curve. 
Proof. Milnor [26, Appendix] showed that a connected one-dimensional $C^{\infty}$ submanifold of $\mathbb{R}^{d}$ is $C^{\infty}$ diffeomorphic to a circle or interval. One can easily check that his proof works for a connected one-dimensional $C^{1}$ submanifold $M \subset \mathbb{R}^{d}$ as well, yielding that it is $C^{1}$ diffeomorphic to a circle or to an interval. Now a standard straightforward argument gives our assertion.

Theorem 8.8. Let $A \subset \mathbb{R}^{d}$ be a closed set with $\operatorname{dim} A=1$. Then, reach $A>0$ if and only if the following two statements hold:

(i) there exists an $L>0$ such that each component of $A$ is a singleton or a simple or closed simple $C^{1,1}$ curve with parameter $L$;

(ii) the inverse of the embedding $A \hookrightarrow \mathbb{R}^{d}$ is uniformly continuous (with respect to the intrinsic distance in $A$ ), i.e., for any $\varepsilon>0$ there exists $\delta>0$ such that

$$
|x-y|<\delta \Longrightarrow d_{A}(x, y)<\varepsilon, \quad x, y \in A .
$$

Proof. Assume first that (i) and (ii) hold and let $L>0$ be a parameter from (i). We shall show that there exists $\rho>0$ such that

$$
\operatorname{dist}(y-x, \operatorname{Tan}(A, x)) \leq \frac{|y-x|^{2}}{2 \rho}, \quad x, y \in A,
$$

which is equivalent to reach $A \geq \rho$ (see Proposition 3.3).

Choose a $\delta>0$ which corresponds by (ii) to $\varepsilon:=1 /(2 L)$ and set $\rho:=\min \{\delta / 2,(1 /(8 L)\}$. To prove (48), consider two arbitrary different points $x, y \in A$.

If $|y-x| \geq \delta$ then we get dist $(y-x, \operatorname{Tan}(A, x)) \leq|y-x| \leq|y-x|^{2} / \delta$, and (48) follows since $\delta \geq 2 \rho$.

If $|y-x|<\delta$ then $d_{A}(x, y)<1 /(2 L)$ by the choice of $\delta$. Consequently $x$ and $y$ belong to the same component $C$ of $A$. Using (i), it is easy to show that we can choose an arc-length $C^{1,1}$ parametrization $\gamma: I \rightarrow \mathbb{R}^{d}$ of $C$ with parameter $L$ and points $s, t \in I$ such that $x=\gamma(s), y=\gamma(t)$ and $|t-s|=d_{A}(x, y)$ (this is not quite obvious only if $C$ is a simple closed curve). Then $|t-s|<1 /(2 L)$ and so (47) holds. Since $4 L \leq 1 /(2 \rho)$ and $\gamma^{\prime}(s)(t-s) \in \operatorname{Tan}(A, x)$, we see that (47) implies (48).

Now we prove the second implication. Assume that reach $A>0$, fix some $0<$ $\rho<\operatorname{reach} A$, let $x \in R$ be a point of the regular part of $A, R=A \backslash A^{(0)}$, and denote $W:=\operatorname{Tan}(A, x)$ (this is a one-dimensional space by (11)). Proposition 7.4 yields that there exist an open neighbourhood $U$ of $x, c \in W, r>0$ and $C^{1}$ mapping

$$
\varphi: B(c, r) \cap W \rightarrow W^{\perp}
$$

with $(m / \rho)$-Lipschitz derivative (where $m$ is an absolute constant) such that $c+$ $\varphi(c)=x$ and, denoting $V:=B(c, r) \cap W$,

$$
\{t+\varphi(t): t \in V\}=A \cap U .
$$

Without any loss of generality we will suppose that $W=\operatorname{span}\left\{e_{1}\right\}$ and identify in the usual way $W$ with $\mathbb{R}$ and $W^{\perp}$ with $\mathbb{R}^{d-1}$. Now it is easy to see that $A \cap U$ is a simple $C^{1}$ curve with parametrization

$$
\psi: t \mapsto(t, \varphi(t)), \quad t \in V=(c-r, c+r) .
$$

Moreover, $\phi:=\psi^{-1}$ is clearly a local $C^{1}$ chart of $A$ (in the sense of Definition 8.3) and $\left|\psi^{\prime}\right| \leq 1$.

Consider now a point $x \in A^{(0)} \backslash \operatorname{isol} A$. Then, Tan $(A, x)$ is a ray determined by a unit vector $u$, and Lemma 3.9 shows that reach $\left(A \cup\left[x, x-\frac{\rho}{4} u\right]\right) \geq \rho / 4$. Clearly, $x$ 
belongs to the regular part of $A \cup\left[x, x-\frac{\rho}{4} u\right]$ and the above consideration shows that there exists an open neighbourhood $U$ of $x$ and a $C^{1}$ diffeomorphism $\psi$ of an open interval $V$ onto $\left(A \cup\left[x, x-\frac{\rho}{4} u\right]\right) \cap U$ such that $\left|\psi^{\prime}\right| \geq 1$ and $\psi^{\prime}$ is $(4 m / \rho)$-Lipschitz. Moreover, $J:=\psi^{-1}(A)$ is a half-open interval, and $\psi \uparrow_{J}$ is a $C^{1}$ diffeomorphism of $J$ onto $A \cap U$, hence, its inverse is a local $C^{1}$ chart of $A$.

Summarizing both cases, to any point $x \in A \backslash$ isol $A$ there exists a neighbourhood $U_{x}$, open or half-open interval $J_{x}$ and $C^{1}$ diffeomorphism $\psi_{x}: J_{x} \rightarrow A \cap U_{x}$ such that $\psi_{x}^{\prime}$ is $(4 m / \rho)$-Lipschitz and $\left|\psi_{x}^{\prime}\right| \geq 1$. In particular, $\psi_{x}^{-1}$ is a local $C^{1}$ chart of $A$, hence, $A \backslash$ isol $A$ is a one-dimensional $C^{1}$ submanifold with boundary. By Lemma 8.7, any connected component $C$ of $A$ which has more than one point must be a $C^{1}$ simple or closed simple curve. Let $\gamma: I \rightarrow \mathbb{R}^{d}$ be a corresponding $C^{1}$ arc-length parametrization of $C$ (injective on int $I$ ). If $x \in C$, choose $U_{x}, J_{x}$ and $\psi_{x}$ as above. Since $\psi_{x}$ parametrizes some relative neighbourhhood of $x$ in $C$, using Remark 8.5 we obtain that $\gamma$ has locally $L$-Lipschitz derivative with $L:=8 \mathrm{~m} / \rho$. This clearly implies that $\gamma^{\prime}$ is globally $L$-Lipschitz and so condition (i) holds.

It remains to verify condition (ii). Set $\delta:=\min \{\rho / 2,1 /(4 L)\}$. Take two different points $x, y \in A$ at distance $|y-x|<\delta$. Using Lemma 3.4 we get that $A \cap B_{x, y}$ is connected, where $B_{x, y}$ is the closed ball of diameter $|y-x|$ containing $x$ and $y$. Thus, $x$ and $y$ lie in one connected component $C$ of $A$, which is (by already proved condition (i)) a simple or closed simple $C^{1,1}$ curve with parameter $L$. Since $A \cap B_{x, y}$ is connected, we have that $A \cap B_{x, y}=C \cap B_{x, y}$ and we easily see that there exists a $\gamma:[s, t] \rightarrow C \cap B_{x, y}$ which is a simple $C^{1,1}$ arc-length parametrization with parameter $L$ (of a simple subarc of $C$ ) such that $\gamma(s)=x$ and $\gamma(t)=y$. If $s_{1}:=s+1 /(2 L) \in[s, t]$ then, using (46), we get

$$
\left|\gamma\left(s_{1}\right)-x\right| \geq \frac{1}{2}\left|s_{1}-s\right|=\frac{1}{4 L}>|y-x|,
$$

hence, $\gamma\left(s_{1}\right) \notin B_{x, y}$. Consequently, $|t-s| \leq 1 /(2 L)$ and, using (46) again, we get $d_{A}(x, y) \leq|t-s| \leq 2|y-x|$. This clearly proves (ii) and the proof is complete.

We say that a simple $C^{1,1}$ curve $A \subset \mathbb{R}^{d}$ has the quasi-arc property, provided that

(Q) For each $\varepsilon>0$ there exists $\delta>0$ such that $\left|x_{1}-x_{2}\right|<\varepsilon$ whenever $\left\{x_{1}, x_{2}, x_{3}\right\} \subset A,\left|x_{1}-x_{3}\right|<\delta$ and $x_{1}$ and $x_{3}$ belong to different components of $A \backslash\left\{x_{2}\right\}$.

Corollary 8.9. Let $A \subset \mathbb{R}^{d}$ be a closed connected set with $\operatorname{dim} A=1$. Then, reach $A>0$ if and only if it is a $C^{1,1}$ curve of one of the following four types:

(a) $A$ is a simple $C^{1,1}$ arc,

(b) $A$ is a closed simple $C^{1,1}$ curve,

(c) $A$ is a simple $C^{1,1}$ curve homeomorphic to $[0, \infty)$ and with the quasi-arc property,

(d) $A$ is a simple $C^{1,1}$ curve homeomorphic to $\mathbb{R}$ and with the quasi-arc property.

Proof. If $A$ has positive reach then, by Theorem 8.8 it is a simple or closed simple $C^{1,1}$ curve. If (a) or (b) holds, we are done. In the opposite case we can choose a $C^{1,1}$ arc-length parametrization $\gamma: I \rightarrow A$, which is a homeomorphism between $I$ and $A$ and $I$ is not compact (since (a) does not hold). Now observe that $I$ is a closed set. Indeed, assume for the contrary that there exists a point $t \in \bar{I} \backslash I$, 
and let $t_{i} \in I$ be such that $t_{i} \rightarrow t, i \rightarrow \infty$. Since $\gamma$ is 1-Lipschitz, there exists $\lim \gamma\left(t_{i}\right)=: x \in \mathbb{R}^{d}$, and we get $x \in A$ from the closedness of $A$. Since $\gamma^{-1}$ is continuous, we get $t_{i} \rightarrow \gamma^{-1}(x) \in I$, which is a contradiction. So, since $I$ is not compact, $I$ is either isometric with $[0, \infty)$, or equal to $(-\infty, \infty)$. We shall verify property (Q). Let $\varepsilon>0$ be given, set $\delta:=\min \left\{\frac{\varepsilon}{2}, \frac{1}{2}\right.$ reach $\left.A\right\}$ and let $x_{1}, x_{2}, x_{3} \in A$ be such that $\left|x_{1}-x_{3}\right|<\delta$ and $x_{1}, x_{3}$ belong to different components of $A \backslash\left\{x_{2}\right\}$. Using Lemma 3.4 we get that $A \cap \bar{B}\left(x_{1}, \delta\right)$ is connected. Hence, $\left|x_{2}-x_{1}\right| \leq \delta<\varepsilon$, which proves $(\mathrm{Q})$.

To prove the other implication, suppose that $A$ is of a type (a)-(d). Then (i) from Theorem 8.8 trivially holds and so, by Theorem 8.8 , it is sufficient to verify property (ii) from this theorem. If $A$ is a curve of type (a) or (b), it must satisfy property (ii) (indeed, it is easy to show that in these cases, the inverse to the embedding $A \hookrightarrow \mathbb{R}^{d}$ is continuous, and the uniform continuity follows from the compactness of $A)$.

So suppose that $A$ is of type (c) or (d), we have to verify condition (ii). To this end, let $\gamma: I \rightarrow A$ be an arc-length $C^{1,1}$ parametrization of $A$ with parameter $L$ which is a homeomorphism between $I$ and $A$. Note that clearly $\left|t_{1}-t_{2}\right|=$ $d_{A}\left(\gamma\left(t_{1}\right), \gamma\left(t_{2}\right)\right)$ whenever $t_{1}, t_{2} \in I$.

Given $\varepsilon_{0}:=1 /(8 L)$, find $\delta_{0}>0$ by condition (Q). Now, for arbitrary $\varepsilon>0$, put $\delta:=\min \left\{\varepsilon / 2, \delta_{0}\right\}$. It is sufficient to prove that $\left|t_{1}-t_{2}\right|<\varepsilon$ whenever $0 \leq t_{1}<t_{2}$ and $\left|\gamma\left(t_{1}\right)-\gamma\left(t_{2}\right)\right|<\delta$. Suppose, to the contrary, that $0 \leq t_{1}<t_{2},\left|\gamma\left(t_{1}\right)-\gamma\left(t_{2}\right)\right|<\delta$ and $\left|t_{1}-t_{2}\right| \geq \varepsilon$. Then we have $\left|t_{1}-t_{2}\right| \geq 1 /(2 L)$, since otherwise by (46) $\left|\gamma\left(t_{1}\right)-\gamma\left(t_{2}\right)\right| \geq\left|t_{1}-t_{2}\right| / 2 \geq \varepsilon / 2 \geq \delta$. So, setting $x_{2}:=\gamma\left(t_{1}+1 /(4 L)\right)$, we clearly have that $x_{1}:=\gamma\left(t_{1}\right)$ and $x_{3}:=\gamma\left(t_{2}\right)$ belong to different components of $A \backslash\left\{x_{2}\right\}$ and so $\left|x_{1}-x_{2}\right|<\varepsilon_{0}=1 /(8 L)$ by the choice of $\delta_{0}$. On the other hand, by (46) we obtain $\left|x_{1}-x_{2}\right| \geq(1 / 2)(1 /(4 L))=1 /(8 L)$, a contradiction.

Remark 8.10. We have shown that in case (c), $A$ admits a homeomorphic arc-length parametrization $\gamma:[0, \infty) \rightarrow A$. Moreover, we have $\lim _{t \rightarrow \infty}|\gamma(t)|=\infty$. Indeed, otherwise there exists a sequence $t_{i} \rightarrow \infty$ such that $\gamma\left(t_{i}\right) \rightarrow x \in \mathbb{R}^{d}$. Since $A$ is closed, we have $x \in A$. Using the continuity of $\gamma^{-1}$, we get $t_{i} \rightarrow \gamma^{-1}(x) \in I$, which is a contradiction.

Similarly, $\lim _{t \rightarrow \pm \infty}|\gamma(t)|=\infty$ in case (d).

Example 8.11. Set $\psi(t):=\left(t^{2}, t e^{-t^{2}}\right), t \in \mathbb{R}$. Using Remark 8.5, it is easy to check that the image of $\psi$ is a simple $C^{1,1}$ curve (and it is also a one-dimensional $C^{1,1}$ manifold). However, it has neither the quasi-arc property, nor positive reach.

\section{REFERENCES}

[1] G. Alberti, On the structure of singular sets of convex functions, Calc. Var. Partial Differential Equations 2 (1994), 17-27.

[2] G. Alberti, L. Ambrosio and P. Cannarsa, On the singularities of convex functions, Manuscripta Math. 76 (1992), 421-435.

[3] N. Aronszajn, Differentiability of Lipschitzian mappings between Banach spaces, Studia Math. 57 (1976), 147-190.

[4] V. Bangert, Sets with positive reach, Arch. Math. (Basel) 38 (1982), 54-57.

[5] D. Burago, Y. Burago and S. Ivanov, A Course in Metric Geometry, Amer. Math. Soc., Providence, 2001.

[6] F. Clarke, Optimization and Nonsmooth Analysis, SIAM, Philadelphia, 1990.

[7] A. Colesanti and C. Pucci, Qualitative and quantitative results for sets of singular points of convex bodies, Forum Math. 9 (1997), 103-125. 
[8] G. Colombo and L. Thibault, Prox-regular sets and applications, In: Handbook of Nonconvex Analysis and Applications, D.Y. Gao and D. Motreanu Eds., International Press, Boston, 2010, pp. 99-182.

[9] G. Crasta and I. Fragala, On the characterization of some classes of proximally smooth sets, ESAIM: Control, Optimisation and Calculus of Variations (to appear), DOI: $10.1051 / \mathrm{cocv} / 2015022$.

[10] A. Colesanti and D. Hug, Hessian measures of semi-convex functions and applications to support measures of convex bodies, Manuscripta Math. 101 (2000), 209-238.

[11] P. Cannarsa and C. Sinestrari, Semiconcave Functions, Hamilton-Jacobi Equations, and Optimal Control, Progress in Nonlinear Differential Equations and their Applications 58, Birkhäuser, Boston, 2004.

[12] J. Dalphin, Some characterizations of a uniform ball property, Congres SMAI 2013, 437-446, ESAIM Proc. Surveys 45, EDP Sci., Les Ulis, 2014.

[13] J. Dieudonné, Foundations of Modern Analysis, Pure and Applied Mathematics 10-I, Academic Press, New York and London, 1969.

[14] J. Duda, Curves with finite turn, Czechoslovak Math. J. 58(133) (2008), 23-49.

[15] J. Duda and L. Zajíček, Semiconvex functions: representations as suprema of smooth functions and extensions, J. Convex Analysis 16 (2009), 239-260.

[16] J. Duda and L. Zajíček, On vector-valued curves that allow a $C^{1, \alpha}$-parametrization, Acta Math. Hung. 127 (2010), 85-111.

[17] H.G. Eggleston, Notes on Minkowski geometry (I): Relations between the circumradius, diameter, inradius and minimal width of a convex set, J. London Math. Soc. 33 (1958), 76-81.

[18] H. Federer, Curvature measures, Trans. Amer. Math. Soc. 93 (1959), 418-491.

[19] J.H.G. Fu, Tubular neighborhoods in Euclidean spaces, Duke Math. J. 52 (1985), 1025-1046.

[20] P. Hájek and M. Johanis, Smooth Analysis in Banach Spaces, De Gruyter Series in Nonlinear Analysis and Applications 19, De Gruyter, Berlin, 2014

[21] A. Hatcher, Algebraic Topology, Cambridge Univ. Press, Cambridge, 2002.

$[22]$ D. Hug, Generalized curvature measures and singularities of sets with positive reach, Forum Math. 10 (1998), 699-728.

[23] D. Hug, J. Rataj and W. Weil, A product integral representation of mixed volumes of two convex bodies, Adv. Geom. 13 (2013), 633-662.

[24] M. Johanis, A quantitative version of the converse Taylor theorem: $C^{k, \omega}$-smoothness, Colloq. Math. 136 (2014), 57-64.

[25] A. Lytchak, Almost convex subsets, Geom. Dedicata 115 (2005), 201-218.

[26] J.W. Milnor, Topology from the Differentiable Viewpoint, Princeton, New Jersey, 1997.

[27] D. Pokorný and J. Rataj, Normal cycles and curvature measures of sets with d.c. boundary, Adv. Math. 248 (2013), 963-985.

[28] J. Rataj, Determination of spherical area measures by means of dilation volumes, Math. Nachr. 235 (2002), 143-162.

[29] Yu.G. Reshetnyak, On a generalization of convex surfaces (Russian), Mat. Sb. N.S. 40(82) (1956), 381-398.

[30] R. Schneider, Boundary structure and curvature of convex bodies, In: Contributions to Geometry, J. Tölke and J.M. Wills Eds., Birkhuser, Basel-Boston, 1979, pp. 13-59.

[31] S. Scholtes, On hypersurfaces of positive reach, alternating Steiner formul and Hadwigers Problem, preprint, 2013, arXiv:1304.4179

[32] L. Veselý and L. Zajíček, Delta-convex mappings between Banach spaces and applications, Dissertationes Math. (Rozprawy Mat.) 289 (1989), 52 pp.

[33] L. Zajíček, On the differentiation of convex functions in finite and infinite dimensional spaces, Czechoslovak Math. J. 29 (1979), 340-348.

[34] L. Zajíček, On Lipschitz and D.C. surfaces of finite codimension in a Banach space, Czechoslovak Math. J. 58 (2008), 849-864.

Charles University, Faculty of Mathematics and Physics, Sokolovská 83, 18675 Praha 8, Czech Republic

E-mail address: rataj@karlin.mff.cuni.cz

E-mail address: zajicek@karlin.mff.cuni.cz 